\title{
Spin portal to dark matter
}

\author{
H. Hernández-Arellano ${ }^{\dagger}$ and M. Napsuciale* \\ Departamento de Física, Universidad de Guanajuato, Lomas del Bosque 103, \\ Fraccionamiento Lomas del Campestre, 37150 León, Guanajuato, Mexico \\ S. Rodríguez \\ Facultad de Ciencias Físico-Matemáticas, Universidad Autónoma de Coahuila, \\ Edificio A, Unidad Camporredondo, 25000 Saltillo, Coahuila, Mexico
}

(Received 7 February 2018; published 2 July 2018)

\begin{abstract}
In this work, we study the possibility that dark matter fields transform in the $(1,0) \oplus(0,1)$ representation of the homogeneous Lorentz group. In an effective theory approach, we study the lowest-dimension interacting terms of dark matter with standard model fields, assuming that dark matter fields transform as singlets under the standard model gauge group. There are three dimension-four operators, two of them yielding a Higgs portal to dark matter. The third operator couples the photon and $Z^{0}$ fields to the higher multipoles of dark matter, yielding a spin portal to dark matter. For low mass dark matter $(D)$, the decays $Z^{0} \rightarrow \bar{D} D$ and $H \rightarrow \bar{D} D$ are kinematically allowed and contribute to the invisible widths of the $Z^{0}$ and $H$ bosons. We use experimental results on these invisible widths to constrain the values of the low-energy constants $g_{t}$ (for the spin portal) and $g_{s}, g_{p}$ (for the Higgs portal) for this mass region. We calculate the dark matter relic density in our formalism and, using the above constraints, we find that consistency with the experimental value requires dark matter to have a mass $M>43 \mathrm{GeV}$ in the case of the spin portal and $M>62 \mathrm{GeV}$ for the Higgs portal. For higher mass dark matter $\left(M>M_{H} / 2\right)$, we calculate the velocity averaged cross section for the annihilation of dark matter into $\bar{b} b$ and $\tau^{+} \tau^{-}$and compare with the upper bounds recently reported by Fermi-LAT and DES Collaborations, finding that both portals yield results consistent with the reported upper bounds. Finally, we study direct detection by elastic scattering on nuclei. The Higgs portal yields results consistent with the upper bounds reported recently by the XENON Collaboration. The spin portal can also accommodate this data but requires higher values of the dark matter mass or smaller values of the corresponding coupling.
\end{abstract}

DOI: 10.1103/PhysRevD.98.015001

\section{INTRODUCTION}

The elucidation of the nature of dark matter is one of the most important problems in high energy physics [1]. Dark matter gravitational effects were noticed during the first half of the last century [2], and recent precise measurements of the cosmic background radiation conclude that it accounts for around $26 \%$ [3] of the matter-energy content of the universe. In spite of this, an identification of dark matter properties is still lacking and a lot of experimental effort is presently being pursued in order to directly or

\footnotetext{
*Corresponding author. mauro@fisica.ugto.mx

${ }^{\dagger}$ h.hernandezarellano@ugto.mx

*simonrodriguez@uadec.edu.mx

Published by the American Physical Society under the terms of the Creative Commons Attribution 4.0 International license. Further distribution of this work must maintain attribution to the author(s) and the published article's title, journal citation, and DOI. Funded by SCOAP ${ }^{3}$.
}

indirectly detect dark matter particles, based mainly in the WIMP paradigm [4]. The latter is based on the fact that the proper description of the measured dark matter relic density, $\Omega_{\mathrm{DM}}^{\exp } h^{2}=0.1186 \pm 0.0020[3,5]$, requires dark matter to have annihilation cross sections into standard model particles of the order of those produced by the weak interactions.

From the particle physics side, dark matter is a challenging problem since there is no particle in the standard model which can be identified with dark matter and, although some extensions of the standard model such as supersymmetric models or extra-dimension models have candidates to dark matter, no signal for these particles has been found in the exhaustive search for signals of physics beyond the standard model or direct search for dark matter signals carried out at the LHC during the past few years [6-8].

The problem has also been considered in a model independent way using effective field theories, where the low energy effects of the unknown theory at high energies are considered in a systematic expansion, based on general 
principles. Effective theories for scalar, spin $1 / 2$ fermion and vector dark matter have been proposed [9-23], and several experimental direct searches are motivated by these formalisms [24-31].

The standard model contains spin $1 / 2$ fermions (quarks and leptons), spin 1 bosons (gauge bosons) and a spin 0 boson (the Higgs particle) with the corresponding fields transforming in the $\left(\frac{1}{2}, 0\right) \oplus\left(0, \frac{1}{2}\right),\left(\frac{1}{2}, \frac{1}{2}\right)$ and $(0,0)$ representations of the homogeneous Lorentz group (HLG), respectively, and it is natural that effective theories so far formulated for dark matter consider dark matter transforming in these representations, although some phenomenological work has been done for spin 3/2 dark matter fields transforming in the Rarita-Schwinger representation [32-35].

Recently, the quantum field theory of spin one massive particles transforming in the $(1,0) \oplus(0,1)$ representation of the HLG (spin-one matter fields), was studied in detail in [36], with the field being described by a six-component "spinor," analogous to the four-component Dirac spinor describing spin $1 / 2$ fermions. It was shown there that a consistent quantum field theory of spin-one matter fields requires a constrained dynamics formalism but the constraints are second class and can be solved along Dirac conventional method [37]. In order to solve the constraints, however, we need to know the algebraic structure of a covariant basis for the operators acting in the $(1,0) \oplus(0,1)$ representation space, which was previously worked out in [38]. This basis naturally contains a chirality operator, $\chi$, and spin-one matter fields can be decomposed into chiral components transforming in the $(1,0)$ (right) and $(0,1)$ (left) representations. However, the kinetic term in the free Lagrangian is not invariant under independent chiral transformations, therefore spin-one matter fields cannot have linearly realized chiral gauge interactions, hence they cannot have weak interactions. Nonetheless, it is possible to have vectorlike interactions like $U(1)_{Y}$ or $S U(3)_{c}$ standard model interactions. In addition, spin-one matter fields can have naively renormalizable selfinteractions classified also in [36].

In this work, we study the possibility of a $(1,0) \oplus(0,1)$ spacetime structure for dark matter fields. Clearly, dark matter with standard model charges would give sizable contributions to precision measurements of standard model observables; thus, we assume in this work that dark matter fields transform as singlets of the standard model gauge group.

The paper is organized as follows. In the next section, we briefly review the elements of the quantum field theory of spin one matter fields. In Sec. III, we discuss the leading terms in the effective field theory. In Sec. IV, we study the low mass dark matter case, calculate the decay width for $Z^{0} \rightarrow \bar{D} D$ and $H \rightarrow \bar{D} D$ and find the constraints on the low energy constants from the $Z^{0}$ and Higgs invisible widths. Section V contains an analysis of the dark matter relic density in this formalism, when these constraints are taken into account. The possibility of a higher dark matter mass is explored in the following sections. Section VI is devoted to calculate the velocity averaged annihilation of dark matter into $\bar{b} b$ and $\tau^{+} \tau^{-}$in Milky Way dwarf spheroidal satellite galaxies and to compare with available data from a recent FermiLAT-DES joint analysis. The direct detection of dark matter is considered in Sec. VII, where we calculate the observable $\sigma_{p}$ and compare with the most stringent upper bounds reported recently by the XENON1T Collaboration. Finally, we give our conclusions in Sec. VIII and close with an Appendix with the required trace calculations for operators in the $(1,0) \oplus(0,1)$ representation space.

\section{QUANTUM FIELD THEORY FOR SPIN-ONE MATTER FIELDS: BRIEF REVIEW}

In the standard model, matter is described by Dirac fermions which transform in the $(1 / 2,0) \oplus(0,1 / 2)$ representation of the HLG. Spin-one matter fields are the generalization of Dirac construction to $j=1$, i.e., fields transforming in the $(1,0) \oplus(0,1)$ representation. The basic object is a six-component "spinor" $\psi(x)$ and the corresponding quantum field theory was studied in [36], taking advantage of the general construction of a covariant basis for $(j, 0) \oplus(0, j)$ representation space introduced in [38]. For $j=1$, the covariant basis is given by the set of $6 \times 6$ matrices $\left\{1, \chi, S^{\mu \nu}, \chi S^{\mu \nu}, M^{\mu \nu}, C^{\mu \nu \alpha \beta}\right\}$ where $\chi$ is the chirality operator, $S^{\mu \nu}$ stands for a symmetric traceless $\left(S^{\mu}{ }_{\mu}=0\right)$ matrix tensor transforming in the $(1,1)$ representation of the HLG, $M^{\mu \nu}$ are the HLG generators and $C^{\mu \nu \alpha \beta}$ is a matrix tensor transforming in the $(2,0) \oplus(0,2)$ representation of the HLG.

The spin-one matter field is written as

$$
\begin{aligned}
\psi(x)= & \sum_{\lambda} \int \frac{d^{3} p}{\sqrt{(2 \pi)^{3} 2 E}}\left[a_{\lambda}(p) U(p, \lambda) e^{-i p . x}\right. \\
& \left.+b_{\lambda}^{\dagger}(p) V(p, \lambda) e^{i p . x}\right],
\end{aligned}
$$

where $U(p, \lambda)(V(p, \lambda))$ stands for the particle (antiparticle) solution with polarization $\lambda$, respectively. In contrast with the Dirac case, spin-one matter particle and antiparticle have the same parity. These solutions satisfy

$$
\begin{aligned}
& \sum_{\lambda} U(p, \lambda) \bar{U}(p, \lambda)=\frac{S(p)+M^{2}}{2 M^{2}}, \\
& \sum_{\lambda} V(p, \lambda) \bar{V}(p, \lambda)=\frac{S(p)+M^{2}}{2 M^{2}},
\end{aligned}
$$

where $S(p) \equiv S^{\mu \nu} p_{\mu} p_{\nu}$.

The free Lagrangian for spin-one matter fields is given by

$$
\mathcal{L}=\frac{1}{2} \partial^{\mu} \bar{\psi}(x)\left(g_{\mu \nu}+S_{\mu \nu}\right) \partial^{\nu} \psi(x)-M^{2} \bar{\psi}(x) \psi(x),
$$

where $\bar{\psi}(x) \equiv(\psi(x))^{\dagger} S^{00}$. The $S^{\mu \nu}$ operators satisfy the following anticommutation relations 


$$
\begin{aligned}
\left\{S^{\mu \nu}, S^{\alpha \beta}\right\}= & \frac{4}{3}\left(g^{\mu \alpha} g^{\nu \beta}+g^{\nu \alpha} g^{\mu \beta}-\frac{1}{2} g^{\mu \nu} g^{\alpha \beta}\right) \\
& -\frac{1}{6}\left(C^{\mu \alpha \nu \beta}+C^{\mu \beta \nu \alpha}\right) .
\end{aligned}
$$

Further algebraic relations of the operators in the covariant basis and the connection with the traces needed for the calculations in this work are deferred to an Appendix. The propagator for spin-one matter particles is given by

$$
i \pi(p)=i \frac{S(p)-p^{2}+2 M^{2}}{2 M^{2}\left(p^{2}-M^{2}+i \varepsilon\right)} .
$$

An important outcome of this formalism is that the freefield Lagrangian can be decomposed in terms of the chiral components as

$\mathcal{L}=\frac{1}{2} \partial^{\mu} \overline{\psi_{R}} \partial_{\mu} \psi_{L}+\frac{1}{2} \partial^{\mu} \overline{\psi_{R}} S_{\mu \nu} \partial_{\nu} \psi_{R}-M^{2} \overline{\psi_{R}} \psi_{L}+R \leftrightarrow L$,

where

$$
\psi_{R}=\frac{1}{2}(1+\chi) \psi, \quad \psi_{L}=\frac{1}{2}(1-\chi) \psi .
$$

The right (left) field $\psi_{R}\left(\psi_{L}\right)$ transforms in $(1,0)((0,1))$ representation of the HLG. Notice that in the massless case, the kinetic term couples right and left components; hence, it is not invariant under independent chiral transformations. Therefore, spin-one matter fields cannot have chiral gauge interactions, although they admit vector gauge interactions. Concerning the standard model interactions, spin-one matter fields can have only $U(1)_{Y}$ or $S U(3)_{C}$ gauge interactions but not $S U(2)_{L}$ interactions, or simply be standard model singlets. This result motivate us to explore the possibility that dark matter be described by spin-one matter fields and we start with the simplest and most likely possibility: spin-one dark matter fields transforming as singlets under the standard model gauge group.

\section{DARK MATTER AS SPIN-ONE MATTER FIELDS: EFFECTIVE THEORY}

If we consider dark matter as spin-one matter fields (spin-one dark matter fields in the following) transforming as singlets under the standard model group, dark matter does not feel the standard model charges. On the other side, if we have more than one dark matter field, dark matter can have gauge interactions with its own (vectorlike) dark gauge group. In the following, we will assume a simple $U(1)_{D}$ structure for the dark gauge group, but the generalization of our results to $S U(N)_{D}$ is straightforward. We remark that the only effect of this dark gauge structure in this work is to provide to dark matter particles with dark charges distinguishing particles from antiparticles and preventing the direct decay of a dark matter particle into standard model ones.

At high energies, the standard model and dark sectors couple in a yet unknown way but the low energy effects of such theory can be classified in an expansion in derivatives of the fields. Each term in this expansion has a low energy constant and the importance at low energies of each term depends on the dimension of the corresponding operator, in such a way that the most important effects are given by the lowest-dimension operators.

The Lagrangian must be a complete scalar operator and if dark matter fields are standard model singlets (and standard model fields are singlets of the dark gauge group) the only possibility to have a scalar interacting Lagrangian is that it be composed of products of singlet operators on both sides. The construction of the lowest-dimension interacting operators in this case, requires to classify the singlet operators in both sectors. The most general form of this interaction is

$$
\mathcal{L}_{\text {int }}=\sum_{n} \frac{1}{\Lambda^{n-4}} \mathcal{O}_{\mathrm{SM}} \mathcal{O}_{\mathrm{DM}}
$$

where $\Lambda$ is an energy scale compensating the dimension $n$ of the product of the standard model singlet operators $\mathcal{O}_{\mathrm{SM}}$ constructed with standard model fields and $\mathcal{O}_{\mathrm{DM}}$ made of spin-one dark matter fields.

It is easy to convince oneself that the lowest-dimension standard model singlet operators are $\tilde{\phi} \phi$ and $B_{\mu \nu}$, where $\phi$ stands for the standard model Higgs doublet and $B_{\mu \nu}$ denotes the $U(1)_{Y}$ stress tensor. Indeed, $\tilde{\phi} \phi$ is simply the singlet of the $\mathbf{2} \otimes \mathbf{2}$ product of $S U(2)_{L}$ (and also a singlet under $S U(3)$ and $\left.U(1)_{Y}\right)$, while in general under $S U(N)$ gauge transformations $U(x)$, the stress (matrix) tensor operator transforms as

$$
F^{\mu \nu} \rightarrow U(x) F^{\mu \nu} U^{-1}(x),
$$

being strictly invariant only in the $U(1)$ case; thus, in the standard model, the $U(1)_{Y}$ stress tensor $B_{\mu \nu}$ is a singlet under the standard model gauge group. Singlet operators made of fermion fields or other combinations can also be constructed but they are higher dimension.

For spin-one matter fields with a dark gauge group $U(1)_{D}$, the lowest-dimension operators transforming as standard model and dark gauge group singlets are of the form $\bar{\psi} O \psi$ where $O$ is one of the 36 matrix operators in the covariant basis $\left\{\mathbf{1}, \chi, S^{\mu \nu}, \chi S^{\mu \nu}, M^{\mu \nu}, C^{\mu \nu \alpha \beta}\right\}$. These operators are dimension two and, using the symmetry properties of $S^{\mu \nu}$ and $C^{\mu \nu \alpha \beta}$, it is easy to show that the leading interacting terms in the effective theory are given by

$$
\mathcal{L}_{\text {int }}=\bar{\psi}\left(g_{s} \mathbf{1}+i g_{p} \chi\right) \psi \tilde{\phi} \phi+g_{t} \bar{\psi} M_{\mu \nu} \psi B^{\mu \nu}
$$

with low-energy constants $g_{s}, g_{p}$ and $g_{t}$. There is an effective Higgs portal to dark matter interactions with 


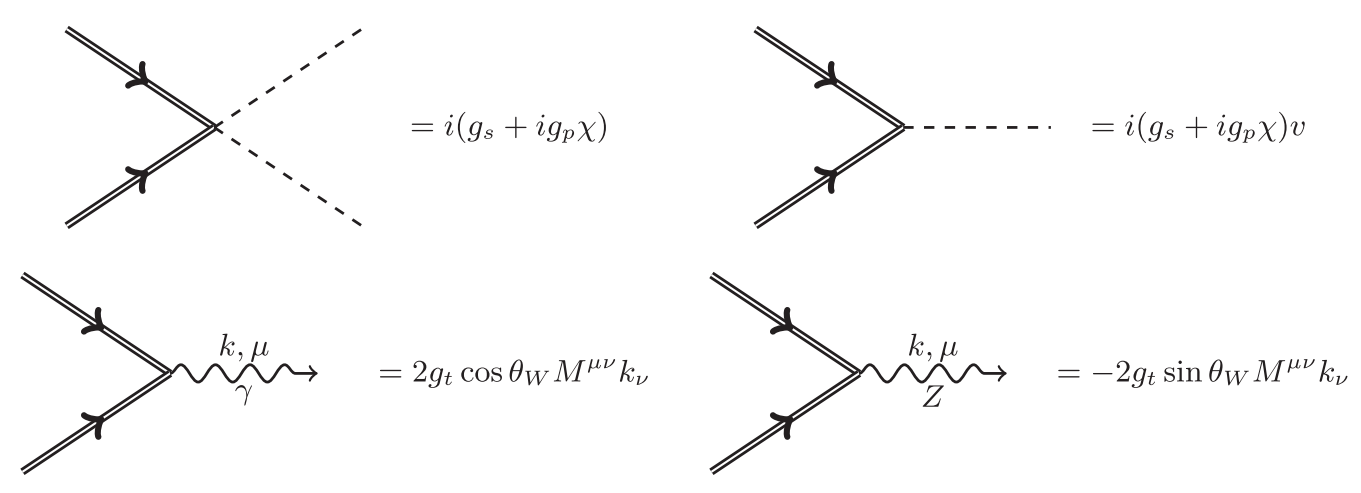

FIG. 1. Feynman rules from the leading terms in the effective theory.

standard model particles given by the first two terms, the second one violating parity. The third term is an effective interaction coupling dark matter to the photon and the $Z^{0}$ boson. Notice that this interaction does not involve the weak charges (operators are standard model singlets), but proceeds through the coupling of the photon and $Z^{0}$ fields to the higher multipoles (magnetic dipole moment and electric quadrupole moment) of the dark matter; thus, we call it the spin portal to dark matter. Since the magnetic moment of a $(1,0) \oplus(0,1)$ field depends on its mass $M$ as $\mu \approx g_{t} / M$ while the electric quadrupole goes as $Q_{E} \approx$ $g_{t} / M^{2}$ [39], the spin portal yields dark matter-standard model interactions suppressed at least as $k / M$ with $k$ denoting the gauge boson (photon or $Z^{0}$ ) momentum. In addition to the interactions in Eq. (10), we have the dimension-four self-interactions described in [36] which are not relevant for the purposes of this paper.

In unitary gauge for the standard model fields, after spontaneous symmetry breaking and diagonalizing the gauge boson sector, we get the following Lagrangian

$$
\begin{aligned}
\mathcal{L}_{\text {int }}= & \frac{1}{2} \bar{\psi}\left(g_{s} \mathbf{1}+i g_{p} \chi\right) \psi(H+v)^{2}+g_{t} \cos \theta_{W} \bar{\psi} M_{\mu \nu} \psi F^{\mu \nu} \\
& -g_{t} \sin \theta_{W} \bar{\psi} M_{\mu \nu} \psi Z^{\mu \nu},
\end{aligned}
$$

where $H$ stands for the Higgs field, $v$ denotes the Higgs vacuum expectation value and $F^{\mu \nu}, Z^{\mu \nu}$ are the electromagnetic and $Z^{0}$ stress tensors, respectively. The Feynman rules arising from the Lagrangian in Eq. (11) are given in Fig. 1.

\section{LIGHT DARK MATTER: $Z^{0} \rightarrow \bar{D} D$ AND $H \rightarrow \bar{D} D$ DECAYS}

The Lagrangian in Eq. (11) induces transitions between the standard model and dark sectors. Annihilation of dark matter into standard model particles such as $\bar{D} D \rightarrow \bar{f} f, \gamma \gamma$, $W^{+} W^{-}, Z^{0} Z^{0}, H H, Z^{0} \gamma, H \gamma, Z^{0} H$ are induced by these interactions under appropriate kinematical conditions. Also, for light dark matter, the decays $Z^{0} \rightarrow \bar{D} D$ and
$H \rightarrow \bar{D} D$ are kinematically allowed and contribute to the invisible $Z^{0}$ and $H$ widths, respectively.

A straightforward calculation yields the following invariant amplitude for the $Z^{0}(k, \epsilon) \rightarrow D\left(p_{1}\right) \bar{D}\left(p_{2}\right)$ decay

$$
\left.-i \mathcal{M}=2 g_{t} S_{W} \bar{U}\left(p_{1}, \lambda_{1}\right) M^{\mu \nu} k_{\nu} V\left(p_{2}, \lambda_{2}\right)\right) \epsilon_{\mu}(k),
$$

where $S_{W}=\sin \theta_{W}$. The calculation of the average squared amplitude can be reduced to a trace of products of operators in the covariant basis of $(1,0) \oplus(0,1)$ representation space, in a procedure similar to conventional calculations with Dirac fermions. We obtain

$$
\begin{aligned}
|\overline{\mathcal{M}}|^{2}= & \frac{4}{3} g_{t}^{2} S_{W}^{2} \operatorname{Tr}\left[\frac{S\left(p_{1}\right)+M^{2}}{2 M^{2}} M^{\mu \nu} \frac{S\left(p_{2}\right)+M^{2}}{2 M^{2}} M^{\alpha \beta}\right] k_{\nu} k_{\beta} \\
& \times\left(-g_{\mu \alpha}+\frac{k_{\mu} k_{\alpha}}{M_{Z}^{2}}\right) .
\end{aligned}
$$

The trace-ology of matrices in $(1,0) \oplus(0,1)$ space is deferred to an Appendix. Using results in the Appendix, we obtain the corresponding decay width as

$\Gamma\left(Z^{0} \rightarrow \bar{D} D\right)=\frac{g_{t}^{2} S_{W}^{2}}{24 \pi M^{4}}\left(M_{Z}^{2}-4 M^{2}\right)^{3 / 2}\left(M_{Z}^{2}+2 M^{2}\right)$.

The invisible width $\Gamma_{\exp }^{\mathrm{inv}}(Z)=499.0 \pm 1.5 \mathrm{MeV}$ reported by the Particle Data Group [5], includes the decay to $\nu \bar{\nu}$. We calculate this decay as

$$
\begin{aligned}
\Gamma\left(Z^{0} \rightarrow \bar{\nu} \nu\right) & \equiv \sum_{i} \Gamma\left(Z^{0} \rightarrow \bar{\nu}_{i} \nu_{i}\right) \\
& =\sum_{i, \alpha} U_{i \alpha}^{2} \frac{M_{Z}^{2}}{24 \pi v^{2}} \sqrt{M_{Z}^{2}-4 m_{\nu_{i}}^{2}} \\
& =\frac{M_{Z}^{3}}{8 \pi v^{2}}=\frac{\sqrt{2} G_{F} M_{Z}^{3}}{8 \pi},
\end{aligned}
$$

where in the last step we neglected the neutrino masses and used the unitarity of the PMNS matrix elements. The Particle Data Group report the value $M_{Z}=91.1876 \pm$ $0.0021 \mathrm{GeV}$ while the $\mu$-Lan Collaboration reported 
the most precise measurement of the Fermi constant as $G_{F}=1.1663788(6) \times 10^{-5} \mathrm{GeV}^{-2}$ [40]. Using these values, we get

$$
\Gamma\left(Z^{0} \rightarrow \bar{\nu} \nu\right)=497.64 \pm 0.03 \mathrm{MeV} .
$$

Subtracting this quantity from the PDG reported value for the invisible width, we get the constraint $\Gamma(Z \rightarrow \bar{D} D)<$ $\Gamma_{Z}^{\mathrm{inv}} \equiv \Gamma_{\exp }^{\mathrm{inv}}(Z)-\Gamma(Z \rightarrow \bar{\nu} \nu)=1.4 \pm 1.5 \mathrm{MeV}$. This width depends on the coupling $g_{t}$ and the dark matter mass $M$, hence the invisible $Z^{0}$ width constrain these parameters to the region shown in Fig. 2.

Similar calculations for the $H \rightarrow \bar{D} D$ decay yield the following decay width

$$
\begin{aligned}
\Gamma(H \rightarrow \bar{D} D)= & \frac{v^{2}}{32 \pi M_{H}^{2} M^{4}} \sqrt{M_{H}^{2}-4 M^{2}} \\
& \times\left[g_{s}^{2}\left(M_{H}^{2}\left(M_{H}^{2}-4 M^{2}\right)+6 M^{4}\right)\right. \\
& \left.+g_{p}^{2} M_{H}^{2}\left(M_{H}^{2}-4 M^{2}\right)\right] .
\end{aligned}
$$

The $H \rightarrow \bar{D} D$ width depends on the unknown $g_{s}, g_{p}$ couplings and on the dark matter mass. This channel contributes to the invisible Higgs width which has been recently reported in $[5,41]$ as $\Gamma_{H}^{\text {inv }}=1.14 \pm 0.04 \mathrm{MeV}$. In this case, the contribution of the $\nu \bar{\nu}$ channel is negligible. The constraints on $g_{s}, g_{p}$ arising from the $\Gamma(H \rightarrow \bar{D} D)<$ $\Gamma_{H}^{\mathrm{inv}}$ condition are also shown in Fig. 2. The solid lines correspond to the central values and the shadow regions to the one sigma regions. We conclude from this plot that the coupling of the spin portal $g_{t}$ in general can be larger than those of the Higgs portal $g_{s}$ or $g_{p}$, by at least one order of magnitude.

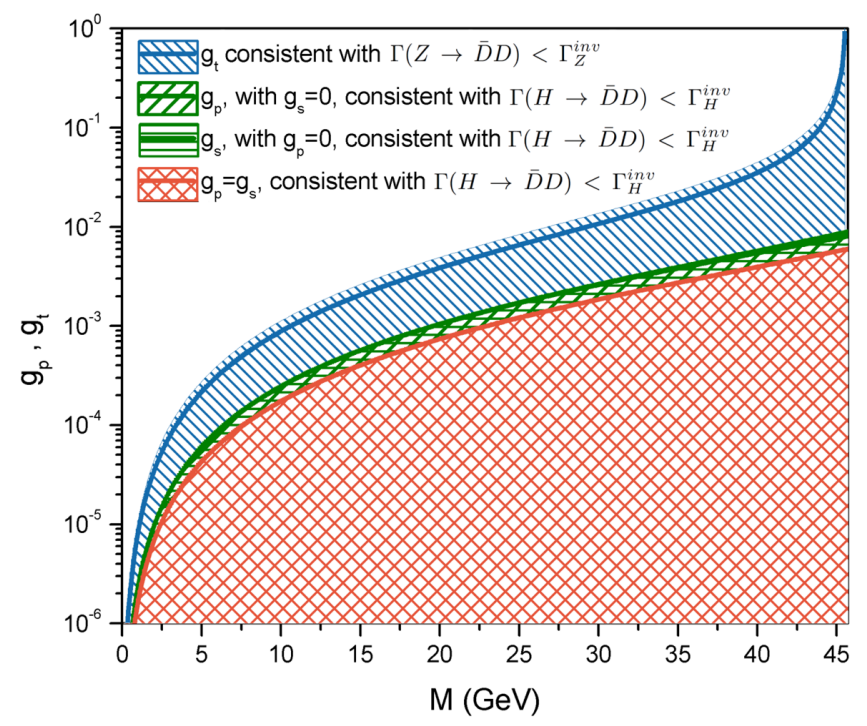

FIG. 2. Parameter space for $g_{t}, g_{s}$ and $g_{p}$ consistent $\Gamma(Z \rightarrow \bar{D} D)<\Gamma_{Z}^{\text {inv }}=1.4 \pm 1.5 \mathrm{MeV} \quad$ and $\Gamma(H \rightarrow \bar{D} D)<$ $\Gamma_{H}^{\text {inv }}=1.14 \pm 0.04 \mathrm{MeV}$ for $M<M_{Z} / 2$. Solid lines correspond to the central values of the invisible decay widths.

\section{DARK MATTER RELIC DENSITY}

\section{A. Boltzman equation}

The evolution of the dark matter comoving number density $n_{D}(T)$ is described by the Boltzmann equation [42]

$$
\frac{d Y}{d x}=-\frac{\lambda(x)}{x^{2}}\left(Y^{2}-Y_{\mathrm{eq}}^{2}\right)
$$

where $x=M / T, Y(x)=n_{D}(x) / T^{3}$ and

$$
\lambda(x) \equiv \frac{M^{3}\left\langle\sigma v_{r}\right\rangle}{H(M)} .
$$

Here, $H(M)=M^{2} \sqrt{\frac{8 \pi^{3} G_{N} g_{*}(M)}{90}}$ stands for the Hubble parameter at the dark mass scale, $M$, with $G_{N}=$ $6.70861(31) \times 10^{-39} \mathrm{GeV}^{-2}$ denoting the Newton gravitational constant [5], $g^{*}(M)$ standing for the relativistic effective degrees of freedom at $T=M$ in the thermal bath and

$$
\begin{aligned}
Y_{\mathrm{eq}}(x) & =\frac{n_{D}^{\mathrm{eq}}}{T^{3}}=\frac{g_{D}}{T^{3}} \int \frac{d^{3} p}{(2 \pi)^{3}} \frac{1}{e^{\frac{E}{T}}-1} \\
& =\frac{3}{2 \pi^{2}} \int_{x}^{\infty} \frac{u \sqrt{u^{2}-x^{2}} d u}{e^{u}-1} \\
& \approx \frac{3}{2 \pi^{2}} \int_{x}^{\infty} e^{-u} u \sqrt{u^{2}-x^{2}} d u .
\end{aligned}
$$

The thermal average $\left\langle\sigma v_{r}\right\rangle$ includes all channels for the annihilation $D\left(p_{1}\right) \bar{D}\left(p_{2}\right) \rightarrow X\left(p_{3}\right) Y\left(p_{4}\right)$ of dark matter into standard model particles $X, Y$ in the thermal bath, and it is given by

$$
\left\langle\sigma v_{r}\right\rangle=\frac{1}{n_{D}^{\mathrm{eq}} n_{\bar{D}}^{\mathrm{eq}}} \int \frac{g_{D} d^{3} p_{1}}{(2 \pi)^{2}} e^{-E_{1} / T} \int \frac{g_{\bar{D}} d^{3} p_{2}}{(2 \pi)^{2}} e^{-E_{2} / T} \sigma v_{r},
$$

where $g_{D}\left(g_{\bar{D}}\right)$ denotes the number of internal d.o.f of the dark matter particle (antiparticle), $v_{r}$ stands for the dark matter particle-antiparticle relative velocity and $\sigma$ is the conventional cross section for the $D\left(p_{1}\right) \bar{D}\left(p_{2}\right) \rightarrow$ $X\left(p_{3}\right) Y\left(p_{4}\right)$ process.

A qualitative analysis of the solution of Eq. (18) assuming the freeze-out of dark matter at some temperature, which would explain dark matter relic density, shows that dark matter must be nonrelativistic at the time of its decoupling from the cosmic plasma [42]. This is consistent with data on dark matter relic density extracted from precision measurement of the cosmic background radiation $3,5]]$. In this case, it is a good approximation to perform a nonrelativistic expansion of $\left\langle\sigma v_{r}\right\rangle$ keeping only the leading terms in the expansion in powers of $v_{r} \ll 1$. This expansion requires the calculation of the flux for dark matter particles in the thermal bath, which can be written as $[43,44]$ 


$$
F=4 \sqrt{\left(p_{1} \cdot p_{2}\right)^{2}-M^{4}}=2\left(s-M^{2}\right) v_{r}
$$

where $v_{r}$ is related to $s$ as

$$
s=2 M^{2}\left(1+\frac{1}{\sqrt{1-v_{r}^{2}}}\right)=4 M^{2}+M^{2} v_{r}^{2}+\cdots
$$

In the last step, we performed the nonrelativistic expansion for $v_{r} \ll 1$. The cross section $\sigma$ is a function of $s$ thus using Eq. (22) the leading terms in the expansion are

$$
\sigma v_{r}=a+b v_{r}^{2}
$$

and performing the thermal average, we obtain

$$
\left\langle\sigma v_{r}\right\rangle=a+\frac{6 b}{x}
$$

For nonrelativistic and light dark matter, the kinematically allowed channels are $\bar{D} D \rightarrow \bar{f} f$ for fermions with
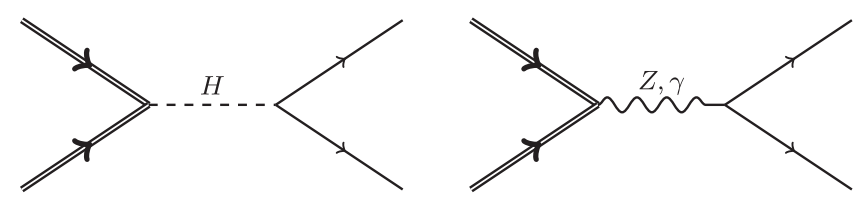

FIG. 3. Feynman diagrams for $\bar{D} D \rightarrow \bar{f} f$.

$m_{f}<M$ and $\bar{D} D \rightarrow \gamma \gamma$. In the following subsection, we calculate the corresponding cross sections in our formalism, perform the nonrelativistic expansion and work out the predictions for the $a, b$ coefficients.

\section{B. Annihilation of dark matter into a fermion-antifermion pair}

There are three contributions to the process $D\left(p_{1}\right) \bar{D}\left(p_{2}\right) \rightarrow f\left(p_{3}\right) \bar{f}\left(p_{4}\right)$ shown in Fig. 3 .

The corresponding amplitudes are given by

$$
\begin{aligned}
-i \mathcal{M}_{H} & =i \frac{m_{f}}{s-M_{H}^{2}} \bar{u}\left(p_{3}\right) v\left(p_{4}\right) \bar{V}\left(p_{2}\right)\left(g_{s} I+i g_{p} \chi\right) U\left(p_{1}\right), \\
-i \mathcal{M}_{\gamma} & =-\frac{4 Q_{f} g_{t} M_{W} S_{W} C_{W}}{v s} \bar{u}\left(p_{3}\right) \gamma^{\mu} v\left(p_{4}\right) \bar{V}\left(p_{2}\right) M_{\mu \beta}\left(p_{1}+p_{2}\right)^{\beta} U\left(p_{1}\right), \\
-i \mathcal{M}_{Z} & =\frac{g_{t} M_{Z} S_{W}}{v\left(s-M_{Z}^{2}\right)} \bar{u}\left(p_{3}\right) \gamma^{\mu}\left(A_{f}+B_{f} \gamma_{5}\right) v\left(p_{4}\right) \bar{V}\left(p_{2}\right) M_{\mu \beta}\left(p_{1}+p_{2}\right) \beta U\left(p_{1}\right) .
\end{aligned}
$$

Here, $C_{W}=\cos \theta_{W}, Q_{f}$ stands for the fermion charge in units of the proton charge $e$, while the $A_{f}, B_{f}$ factors are related to the corresponding fermion weak isospin $T_{3}^{f}$ as

$$
A_{f}=2 T_{3}^{f}-4 Q_{f} S_{W}^{2}, \quad B_{f}=-2 T_{3}^{f} .
$$

A straightforward calculation yields the following average squared amplitude in terms of the Mandelstam variables:

$$
\begin{aligned}
\left|\overline{\mathcal{M}_{f f}}\right|^{2}= & -\frac{g_{t}^{2} M_{Z}^{2} S_{W}^{2}}{9 M^{4} v^{2}\left(s-M_{Z}^{2}\right)^{2}}\left[4 M^{2}\left(A_{f}^{2}+B_{f}^{2}\right) m_{f}^{4}\left(4 M^{2}-s\right)+4 m_{f}^{2}\left(4 M^{2}-s\right)\left(A_{f}^{2} M^{2}\left(2 M^{2}+s-t-u\right)\right.\right. \\
& \left.+B_{f}^{2}\left(2 M^{4}-M^{2}(s+t+u)-s^{2}\right)\right)+\left(A_{f}^{2}+B_{f}^{2}\right)\left(16 M^{8}-4 M^{6}(s+4(t+u))+4 M^{4}(t+u)(s+t+u)\right. \\
& \left.\left.+M^{2}\left(4 s^{3}-2 s\left(t^{2}+u^{2}\right)\right)+s^{2}\left((t-u)^{2}-s^{2}\right)\right)\right]+\frac{8 A_{f} C_{W} Q_{f} g_{t}^{2} M_{W} M_{Z} S_{W}^{2}}{9 M^{4} s v^{2}\left(s-M_{Z}^{2}\right)}\left[4 M^{2} m_{f}^{2}\left(4 M^{2}-s\right)\left(2 M^{2}+s-t-u\right)\right. \\
& +4 m_{f}^{4}\left(4 M^{4}-M^{2} s\right)+16 M^{8}-4 M^{6}(s+4(t+u))+4 M^{4}(t+u)(s+t+u)+M^{2}\left(4 s^{3}-2 s\left(t^{2}+u^{2}\right)\right) \\
& \left.+s^{2}\left((t-u)^{2}-s^{2}\right)\right]+\frac{4 A_{f} m_{f}^{2} g_{s} g_{t} M_{Z} S_{W}}{9 M^{4} v\left(s-M_{H}^{2}\right)\left(s-M_{Z}^{2}\right)} s\left(2 M^{2}-s\right)(t-u)-\frac{16 C_{W} m_{f}^{2} Q_{f} g_{s} g_{t} M_{W} S_{W}}{9 M^{4} v\left(s-m_{H}^{2}\right)} \\
& -\frac{16 C_{W}^{2} Q_{f}^{2} g_{t}^{2} M_{W}^{2} S_{W}^{2}\left[4 M^{2} m_{f}^{2}\left(4 M^{2}-s\right)(t-u)\right.}{9 M^{4} s^{2} v^{2}}\left[\left(2 M^{2}+s-t-u\right)+4 m_{f}^{4}\left(4 M^{4}-M^{2} s\right)\right. \\
& \left.+16 M^{8}-4 M^{6}(s+4(t+u))+4 M^{4}(t+u)(s+t+u)+M^{2}\left(4 s^{3}-2 s\left(t^{2}+u^{2}\right)\right)+s^{2}\left((t-u)^{2}-s^{2}\right)\right] \\
& +\frac{m_{f}^{2}}{9 M^{4}\left(s-M_{H}^{2}\right)^{2}}\left(s-4 m_{f}^{2}\right)\left[g_{p}^{2} s\left(s-4 M^{2}\right)+g_{s}^{2}\left(6 M^{4}-4 M^{2} s+s^{2}\right)\right] .
\end{aligned}
$$

Integrating the phase space, we obtain the following cross section for $\bar{D} D \rightarrow \bar{f} f$, where we can easily identify the individual contributions from the $H, Z^{0}$, and $\gamma$ exchange as well as the $Z^{0}-\gamma$ interference: 


$$
\begin{aligned}
\sigma_{f f}(s)= & \frac{1}{72 \pi M^{4} \sqrt{s}} \frac{\sqrt{s-4 m_{f}^{2}}}{F}\left[\frac{m_{f}^{2}\left(s-4 m_{f}^{2}\right)\left(g_{p}^{2} s\left(s-4 M^{2}\right)+g_{s}^{2}\left(6 M^{4}-4 M^{2} s+s^{2}\right)\right)}{\left(s-M_{H}^{2}\right)^{2}}\right. \\
& +\frac{2 g_{t}^{2} M_{Z}^{2} S_{W}^{2} s\left(s-4 M^{2}\right)\left(2 M^{2}+s\right)\left(2\left(A_{f}^{2}-2 B_{f}^{2}\right) m_{f}^{2}+s\left(A_{f}^{2}+B_{f}^{2}\right)\right)}{3 v^{2}\left(s-M_{Z}^{2}\right)^{2}} \\
& +\frac{32 C_{W}^{2} Q_{f}^{2} g_{t}^{2} M_{W}^{2} S_{W}^{2}\left(s-4 M^{2}\right)\left(2 M^{2}+s\right)\left(2 m_{f}^{2}+s\right)}{3 v^{2} s} \\
& \left.-\frac{16 A_{f} C_{W} Q_{f} g_{t}^{2} M_{W} M_{Z} S_{W}^{2}\left(s-4 M^{2}\right)\left(2 M^{2}+s\right)\left(2 m_{f}^{2}+s\right)}{3 v^{2}\left(s-M_{Z}^{2}\right)}\right]
\end{aligned}
$$

Notice that the $H-Z$ and $H-\gamma$ interferences vanish after integration of phase space.

\section{Dark matter annihilation into two photons}

This process is induced by the $t$ and $u$ channel dark matter exchange shown in Fig. 4. The corresponding amplitudes are given by

$$
\begin{aligned}
-i \mathcal{M}_{t} & =i \frac{2 g_{t}^{2} C_{W}^{2}}{M^{2}} \bar{V}\left(p_{2}, \lambda_{2}\right) M_{\alpha \beta} \frac{S\left(p_{1}-p_{3}\right)-t+2 M^{2}}{t-M^{2}} M_{\mu \nu} U\left(p_{1}, \lambda_{1}\right) p_{4}^{\alpha} \eta^{\beta}\left(p_{4}\right) p_{3}^{\mu} \epsilon^{\nu}\left(p_{3}\right), \\
-i \mathcal{M}_{u} & =i \frac{2 g_{t}^{2} C_{W}^{2}}{M^{2}} \bar{V}\left(p_{2}, \lambda_{2}\right) M_{\mu \nu} \frac{S\left(p_{1}-p_{4}\right)-u+2 M^{2}}{u-M^{2}} M_{\alpha \beta} U\left(p_{1}, \lambda_{1}\right) p_{4}^{\alpha} \eta^{\beta}\left(p_{4}\right) p_{3}^{\mu} \epsilon^{\nu}\left(p_{3}\right) .
\end{aligned}
$$

The average squared amplitude is given by

$$
\left|\overline{\mathcal{M}_{\gamma \gamma}}\right|^{2}=\left(\frac{2 g_{t}^{2} C_{W}^{2}}{3 M^{2}}\right)^{2} \operatorname{Tr}\left[\frac{S\left(p_{2}\right)+M^{2}}{2 M^{2}} T_{\alpha \beta \mu \nu} \frac{S\left(p_{1}\right)+M^{2}}{2 M^{2}} \bar{T}_{\sigma}^{\beta}{ }^{\nu}\right] p_{3}^{\mu} p_{3}^{\rho} p_{4}^{\alpha} p_{4}^{\sigma},
$$

where

$$
\begin{aligned}
& T_{\alpha \beta \mu \nu}=M_{\alpha \beta} \frac{S\left(p_{1}-p_{3}\right)-t+2 M^{2}}{t-M^{2}} M_{\mu \nu}+M_{\mu \nu} \frac{S\left(p_{1}-p_{4}\right)-u+2 M^{2}}{u-M^{2}} M_{\alpha \beta}, \\
& \bar{T}_{\alpha \beta \mu \nu}=M_{\mu \nu} \frac{S\left(p_{1}-p_{3}\right)-t+2 M^{2}}{t-M^{2}} M_{\alpha \beta}+M_{\alpha \beta} \frac{S\left(p_{1}-p_{4}\right)-u+2 M^{2}}{u-M^{2}} M_{\mu \nu} .
\end{aligned}
$$

A straightforward calculation using the algebraic relations in the Appendix yields

$$
\begin{aligned}
\overline{\left|\mathcal{M}_{\gamma \gamma}\right|^{2}}= & \frac{2 C_{W}^{4} g_{t}^{4}}{9 M^{8}\left(t-M^{2}\right)^{2}\left(u-M^{2}\right)^{2}}\left[6(t u)^{4}+2(t u)^{3}\left(-13 M^{4}+11 M^{2} s+2 s^{2}\right)+(t u)^{2}\left(42 M^{8}-76 M^{6} s+33 M^{4} s^{2}\right.\right. \\
& \left.+4 M^{2} s^{3}+2 s^{4}\right)+2 M^{2} t u\left(-15 M^{10}+43 M^{8} s-44 M^{6} s^{2}+17 M^{4} s^{3}-6 M^{2} s^{4}+2 s^{5}\right) \\
& \left.+M^{4}\left(8 M^{12}-32 M^{10} s+51 M^{8} s^{2}-40 M^{6} s^{3}+25 M^{4} s^{4}-12 M^{2} s^{5}+2 s^{6}\right)\right] .
\end{aligned}
$$

Integrating the final state phase space, we get the following cross section:

$$
\begin{aligned}
\sigma_{\gamma \gamma}(s)= & \frac{1}{F \sqrt{1-\frac{4 M^{2}}{s}}} \frac{C_{W}^{4} g_{t}^{4}}{540 \pi M^{8}}\left[120 M^{4}\left(4 M^{4}-3 M^{2} s-2 s^{2}\right) \tanh ^{-1} \sqrt{1-\frac{4 M^{2}}{s}}\right. \\
& \left.+s \sqrt{1-\frac{4 M^{2}}{s}}\left(-10 M^{6}+228 M^{4} s-99 M^{2} s^{2}+43 s^{3}\right)\right] .
\end{aligned}
$$




\section{Dark matter relic density}

Expanding the $\bar{D} D \rightarrow \bar{f} f$ and $\bar{D} D \rightarrow \gamma \gamma$ cross sections, we get

$$
\sigma v_{r} \equiv \sigma_{\gamma \gamma} v_{r}+\sum_{f} \sigma_{\overline{f f}} v_{r}=a+b v_{r}^{2}
$$

where the sum runs over all the kinematically allowed fermion states $\left(m_{f}<M\right)$ and

$$
\begin{aligned}
a= & \frac{29 C_{W}^{4} g_{t}^{4}}{18 \pi M^{2}}+\sum_{f} \frac{N_{f} g_{s}^{2} m_{f}^{2}\left(M^{2}-m_{f}^{2}\right)^{\frac{3}{2}}}{12 \pi M^{3}\left(M_{H}^{2}-4 M^{2}\right)^{2}}, \\
b= & \frac{365 C_{W}^{4} g_{t}^{4}}{216 \pi M^{2}}+\sum_{f} \frac{N_{f} \sqrt{M^{2}-m_{f}^{2}}}{864 \pi M^{5}}\left(\frac{96 M^{4} g_{t}^{2} M_{Z}^{2} S_{W}^{2}\left(\left(A_{f}^{2}-2 B_{f}^{2}\right) m_{f}^{2}+2 M^{2}\left(A_{f}^{2}+B_{f}^{2}\right)\right)}{v^{2}\left(M_{Z}^{2}-4 M^{2}\right)^{2}}\right. \\
& +\frac{192 A_{f} M^{2} C_{W} Q_{f} g_{t}^{2} M_{W} M_{Z} S_{W}^{2}\left(m_{f}^{2}+2 M^{2}\right)}{v^{2}\left(M_{Z}^{2}-4 M^{2}\right)}+\frac{96 C_{W}^{2} Q_{f}^{2} g_{t}^{2} M_{W}^{2} S_{W}^{2}\left(m_{f}^{2}+2 M^{2}\right)}{v^{2}} \\
& -\frac{6 M^{2} m_{f}^{2}\left(8 g_{p}^{2}\left(4 M^{2}-M_{H}^{2}\right)\left(M^{2}-m_{f}^{2}\right)+g_{s}^{2}\left(-8 m_{f}^{2}\left(M^{2}-M_{H}^{2}\right)-11 M^{2} M_{H}^{2}+20 M^{4}\right)\right)}{\left(M_{H}^{2}-4 M^{2}\right)^{3}} \\
& \left.-\frac{9 M^{2} m_{f}^{2} g_{s}^{2}\left(4 M^{2}-5 m_{f}^{2}\right)}{\left(M_{H}^{2}-4 M^{2}\right)^{2}}\right),
\end{aligned}
$$

with $N_{f}=3$ for quarks and $N_{f}=1$ for leptons.

In Fig. 5, we show the Higgs and spin portal contributions to $\left\langle\sigma v_{r}\right\rangle$ as a function of the couplings for different values of the dark matter mass. Using Eqs. (25), (38), we numerically solve the Boltzman equation (18) for different values the couplings $g_{t}, g_{s}$ and $g_{p}$, matching the solution $Y(x)$ with the equilibrium solution $Y_{\text {eq }}(x)$ in Eq. (20) at high temperatures, i.e., in the relativistic regime $x \ll 1$. The solutions are shown in Fig. 6. Clearly, at some $x_{f}$, the solution $Y(x)$ departs from the equilibrium solution $Y_{\text {eq }}(x)$ and dark matter decouples from the cosmic plasma in the nonrelativistic regime, $x \gg 1$.

In order to find the dark matter relic density, we need to calculate $Y$ for the present temperature $T_{0}$. This can be done from the numeric solution to Boltzman equation for specific values of the couplings and $M$ scanning the parameter space consistent with the measured relic density. It is, however, more illustrative to follow the semianalytic procedure that takes advance of the freeze-out mechanism.

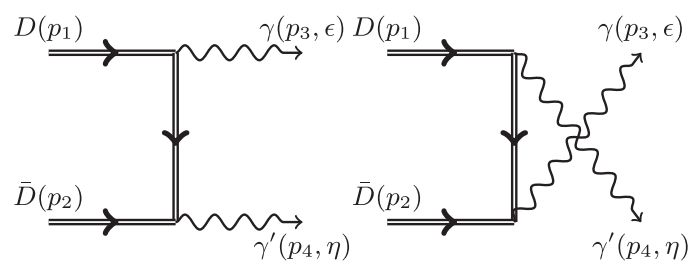

FIG. 4. Feynman diagrams for $\bar{D} D \rightarrow \gamma \gamma$.
For $x>x_{f}$ we have $Y(x) \gg Y_{\text {eq }}(x)$ and we can find an approximate solution neglecting $Y_{\mathrm{eq}}(x)$ in the right-hand side of Eq. (18) and integrating from $T_{f}$ to a given temperature $T$, which for our purposes we take as the present temperature $T_{0}$, to obtain

$$
\frac{1}{Y\left(x_{0}\right)}=\frac{1}{Y\left(x_{f}\right)}+\sqrt{\frac{90}{8 \pi^{3} G_{N}}} M \int_{x_{f}}^{x_{0}} \frac{\left\langle\sigma v_{r}\right\rangle}{\sqrt{g_{*}(x)} x^{2}} d x .
$$

The relic dark matter density is given by

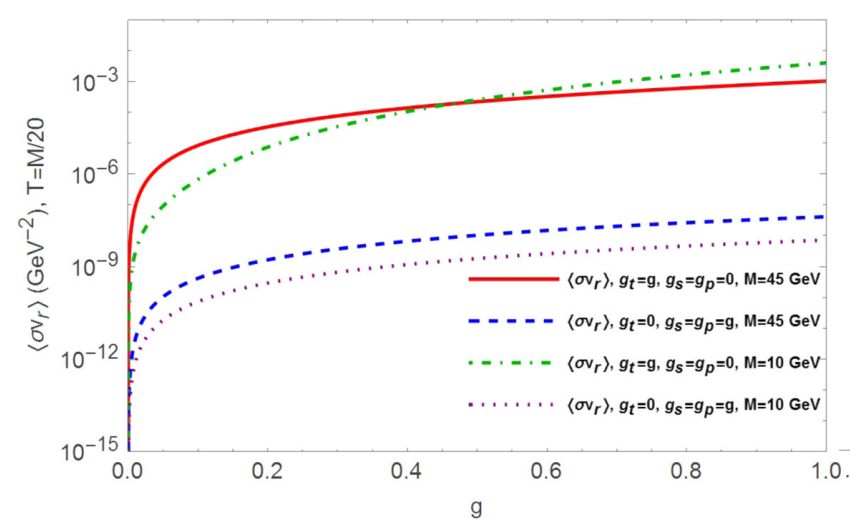

FIG. 5. Individual contributions of the spin portal $\left(g_{t}=g\right.$, $\left.g_{s}=g_{p}=0\right)$ and the Higgs portal $\left(g_{t}=0, g_{s}=g_{p}=g\right)$ to $\left\langle\sigma v_{r}\right\rangle$. Similar results are obtained in the second case when varying independently $g_{s}$ or $g_{p}$. 

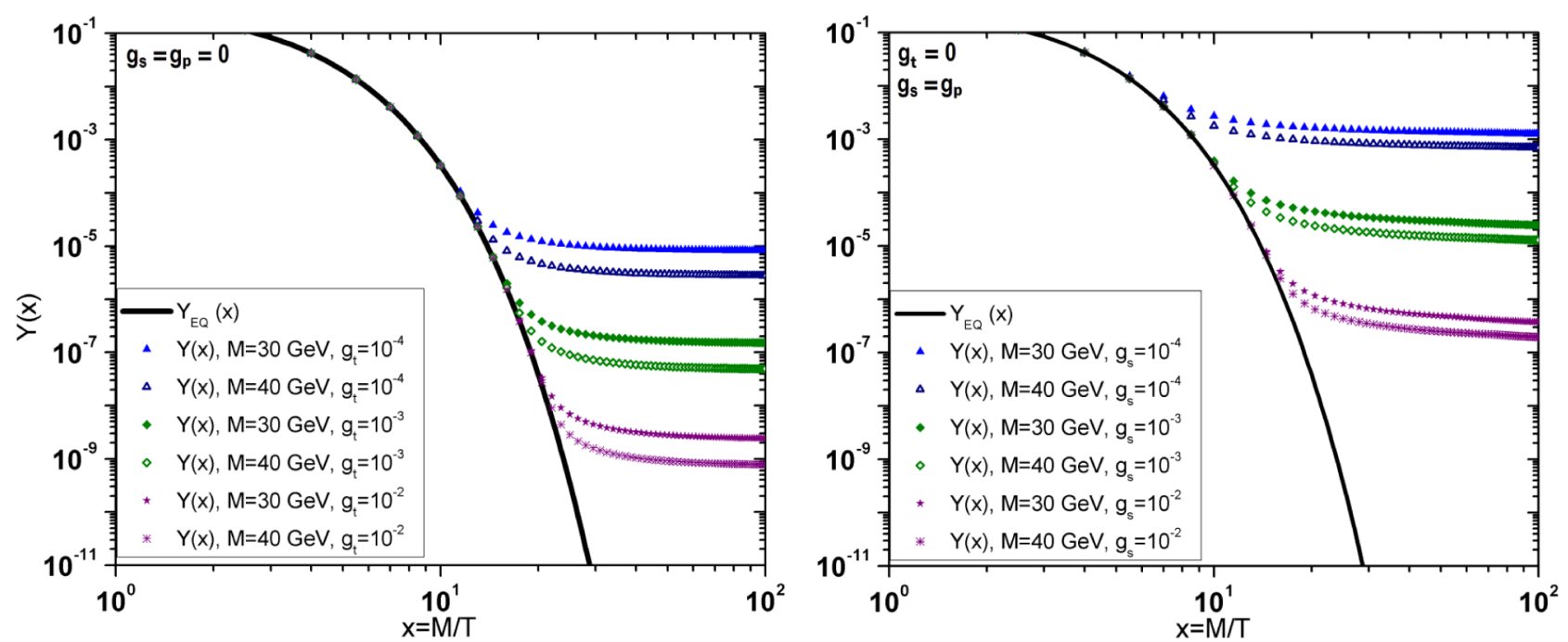

FIG. 6. Solution of the Boltzman equation for the spin portal (left) and Higgs portal (right). Similar results are obtained in the later case when varying independently $g_{s}$ and $g_{p}$. The solid line corresponds to $Y_{\text {eq }}(x)$.

$$
\begin{aligned}
\Omega_{\mathrm{DM}} & =\frac{\rho_{\mathrm{DM}}\left(x_{0}\right)}{\rho_{c}}=\frac{\left(n_{D}\left(x_{0}\right)+n_{\bar{D}}\left(x_{0}\right)\right) M}{\rho_{c}} \\
& =\frac{2 n_{D}\left(x_{0}\right) M}{\rho_{c}}=\frac{2 M Y\left(x_{0}\right) T_{0}^{3}}{\rho_{c}},
\end{aligned}
$$

where we used $n_{\bar{D}}=n_{D}$ and $\rho_{c}=\frac{3 H_{0}^{2}}{8 \pi G_{N}}=1.05371(5) \times$ $10^{-5} h^{2} \mathrm{GeV} / \mathrm{cm}^{3}=8.09619(38) \times 10^{-47} h^{2} \mathrm{GeV}^{4}$ is the critical density [5]. Neglecting the term $Y\left(x_{f}\right)^{-1}$ in Eq. (39) which turns out to be small compared with the second term we get,

$$
\begin{aligned}
\Omega_{\mathrm{DM}} h^{2} & =\frac{2 T_{0}^{3} h^{2}}{\rho_{c}} \sqrt{\frac{8 \pi^{3} G_{N}}{90}}\left(\int_{x_{f}}^{x_{0}} \frac{\left\langle\sigma v_{r}\right\rangle}{\sqrt{g_{*}(x)} x^{2}} d x\right)^{-1} \\
& =4.337 \times 10^{-11} \mathrm{GeV}^{-2}\left(\int_{x_{f}}^{x_{0}} \frac{\left\langle\sigma v_{r}\right\rangle}{\sqrt{g_{*}(x)} x^{2}} d x\right)^{-1},
\end{aligned}
$$

where we used $T_{0}=2.7255(6) K=2.34865(52) \times$ $10^{-13} \mathrm{GeV}$ [5]. Notice that the right-hand side of this equation depends on the couplings and $M$. For a given $M$ we can find the values of the couplings consistent with the measured value of the relic density. In our calculations, we use the complete function $g_{*}(x)$, but our results are quite similar if we use the average over the range of energies considered, $\bar{g}_{*}=33$.

The value of $x_{f}$ can be found from the condition that the annihilation rate equals the expansion rate of the universe

$$
n_{\mathrm{eq}}\left(x_{f}\right)\left\langle\sigma v_{r}\right\rangle\left(x_{f}\right)=H\left(x_{f}\right),
$$

which using the nonrelativistic form for $n_{\text {eq }}(x)$ and Eq. (25) leads to

$$
\left(a+\frac{6 b}{x_{f}}\right) \sqrt{x_{f}} e^{-x_{f}}=\frac{(2 \pi)^{3}}{3 M} \sqrt{\frac{G_{N} g^{*}\left(x_{f}\right)}{90} .}
$$

For the spin portal, the value of $x_{f}$ depends also on $g_{t}$ and $M$, so we have two conditions, Eqs. (41), (43), for the three variables $x_{f}, g_{t}, M$ which are solved numerically to obtain the set of values $g_{t}(M)$ consistent with the measured dark matter relic density. We checked also that these solutions are consistent with the approximations used, i.e., that decoupling occurs when dark matter is nonrelativistic. The values of $x_{f}$ corresponding to $g_{t}(M)$ lie in the range $23.8<x_{f}<27.9$, thus $x_{f} \gg 1$. The set of values $g_{t}(M)$ so obtained, is shown in Fig. 7. Finally, we directly calculate $Y(x)$ from the numeric general solution of the Boltzman equation for the set of values $g_{t}(M)$, matching the solution with $Y_{\mathrm{eq}}(x)$ for $x \ll x_{f}$, finding indeed that $1 / Y\left(x_{f}\right)$ is small compared to $1 / Y\left(x_{0}\right)$ in Eq. (39). A similar procedure is used for the two couplings of the Higgs portal.

In the case of the Higgs portal, we show results for $g_{p}=$ 0 varying $g_{s}$ and $g_{s}=0$ varying $g_{p}$, but similar results are obtained varying both couplings simultaneously. It is clear from Fig. 7 that, for the spin portal, the consistency of the measured relic density with the constraints from data on the $Z^{0}$ invisible width requires a dark matter mass $M \geq 43 \mathrm{GeV}$. Similarly, for the Higgs portal, the consistency of the measured relic density with the constraints imposed by the $H^{0}$ invisible width requires a dark matter mass $M \geq 62 \mathrm{GeV}$. 

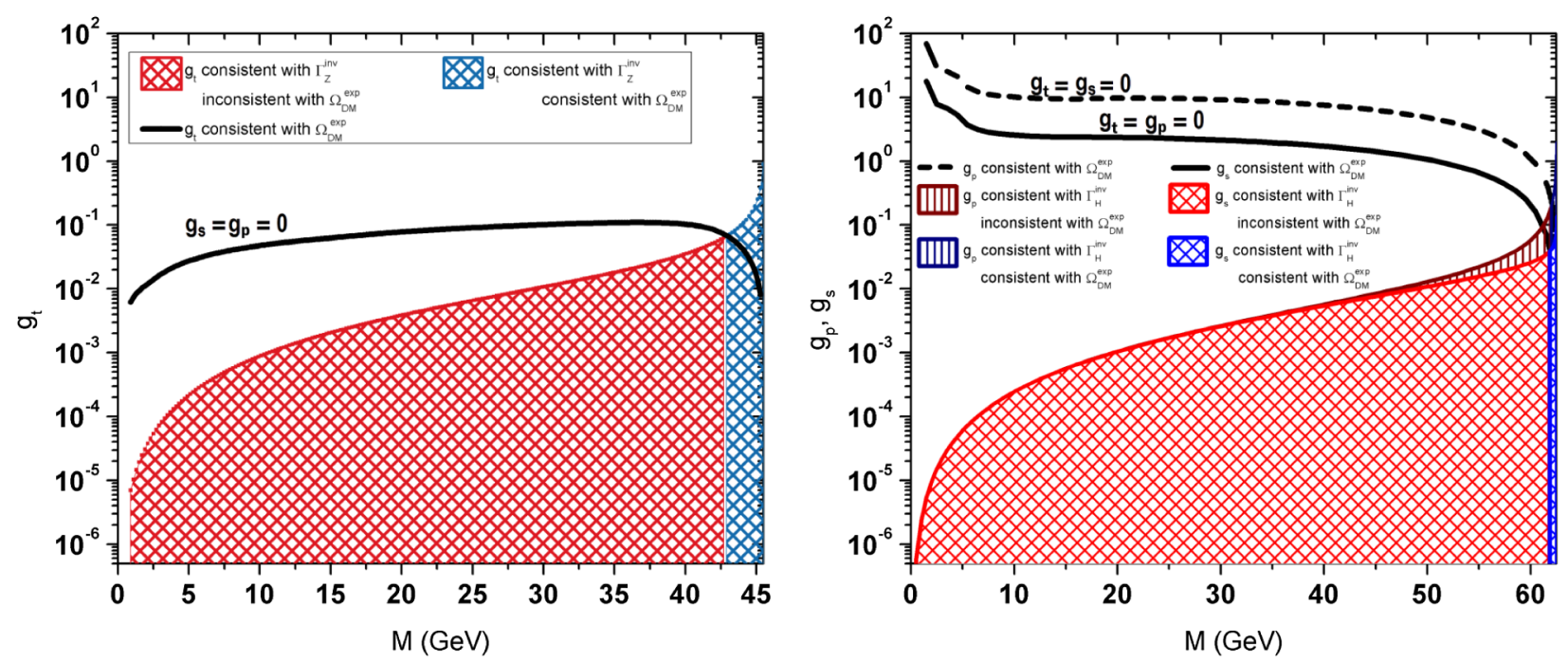

FIG. 7. Values of the couplings consistent with the measured dark matter relic density, $\Omega_{\mathrm{DM}}^{\exp } h^{2}=0.1186 \pm 0.0020$ (solid line), as a function of $M$. The shadowed region in the left panel corresponds to the values consistent with the $Z^{0}$ invisible width, $\Gamma\left(Z^{0} \rightarrow \bar{D} D\right)<\Gamma_{Z}^{\text {inv }}=1.4 \pm 1.5 \mathrm{MeV}$, for the spin portal. These constraints exclude masses below $43 \mathrm{GeV}$ for the spin portal. The shadowed region in the right panel are the values consistent with the constraint $\Gamma(H \rightarrow \bar{D} D)<\Gamma_{H}^{\mathrm{inv}}=1.14 \pm 0.04 \mathrm{MeV}$ for the spin portal. Masses below $62 \mathrm{GeV}$ are excluded for this portal.

\section{DARK MATTER WITH A HIGHER MASS: ANNIHILATION INTO FERMIONS AND FERMILAT-DES DATA}

For dark matter with a mass $M>M_{Z} / 2$ the decay $Z^{0} \rightarrow$ $\bar{D} D$ is kinematically forbidden and we lose the corresponding constraint on the coupling $g_{t}$. Similarly for $M>M_{H} / 2$ the couplings $g_{s}, g_{p}$ are not constrained by the Higgs invisible width. However, there are regions in our universe with high concentrations of dark matter which can be used for its indirect detection by measuring the corresponding annihilation products. In particular, Milky Way dwarf spheroidal satellite galaxies (dSphs) are known to have high dark matter densities and due to their proximity and apparent absence of nonthermal processes are appropriate for this purpose. Recently, the Dark Energy Survey (DES) Collaboration identified eight new candidates for dSphs and in a joint search of gamma ray signals with the Fermi Large Area Telescope (FermiLAT) from dSphs, put stringent bounds on the velocity averaged cross sections for the annihilation of dark matter into $\tau^{+} \tau^{-}$and $\bar{b} b$ in these systems [45].

In our formalism, the cross section for the annihilation of dark matter into $\tau^{+} \tau^{-}$and $\bar{b} b$ is given in Eq. (29) with
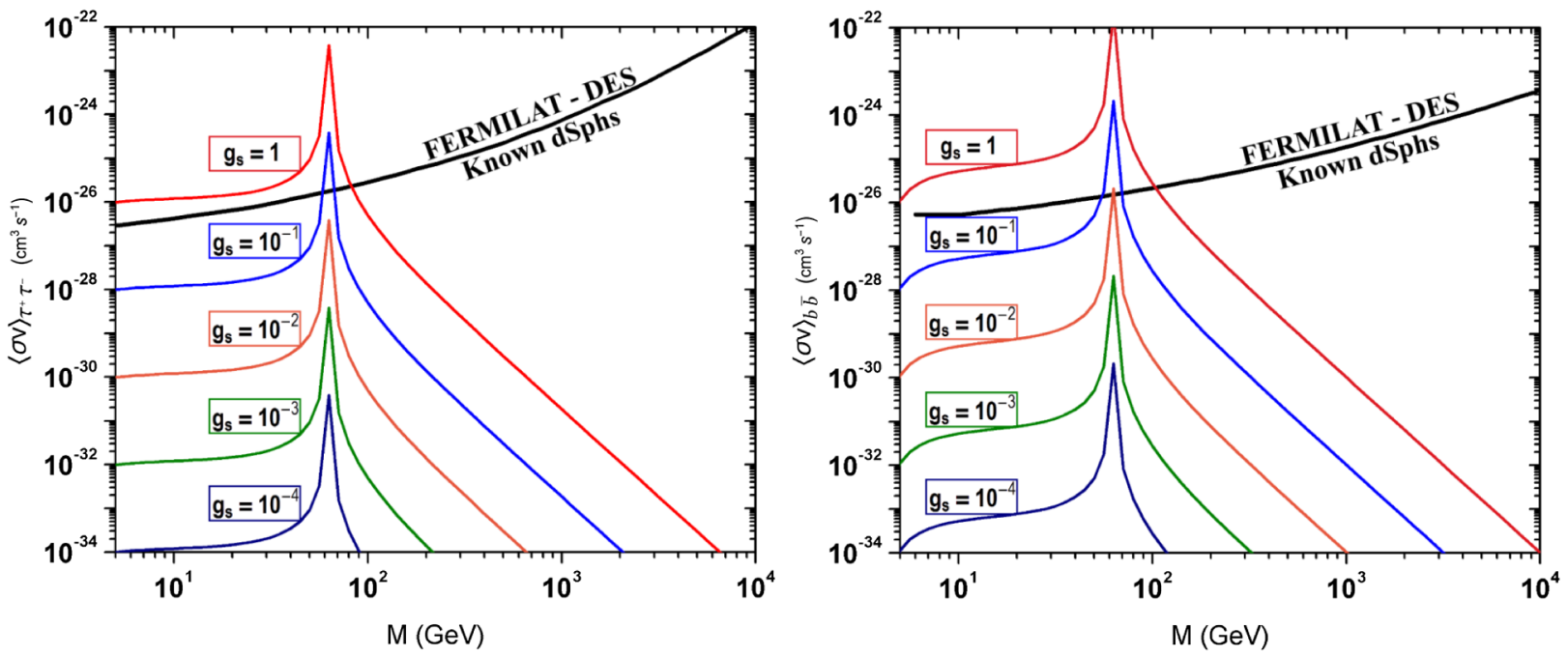

FIG. 8. Velocity averaged cross section for dark matter annihilation into $\tau^{+} \tau^{-}$(left) and $\bar{b} b$ (right) and comparison with Fermilat-DES upper bounds, for different values of $g_{s}$ (Higgs portal). The spin portal yields contributions even smaller and are consistent with these upper bounds. 
$A_{\tau}=-1+4 S_{W}^{2}, B_{\tau}=1$ and $A_{b}=-1+\frac{4}{3} S_{W}^{2}, B_{b}=1$. Since dark matter in the Milky Way halo is still nonrelativistic we take advantage of the nonrelativistic expansion in Eqs. (37), (38) to obtain

$$
\begin{aligned}
\left\langle\sigma v_{r}\right\rangle_{\tau^{+} \tau-} & =\frac{g_{s}^{2} m_{\tau}^{2}\left(M^{2}-m_{\tau}^{2}\right)^{\frac{3}{2}}}{12 \pi M^{3}\left(M_{H}^{2}-4 M^{2}-i \Gamma_{H} M_{H}\right)^{2}}+\mathcal{O}\left(\left\langle v_{r}^{2}\right\rangle\right), \\
\left\langle\sigma v_{r}\right\rangle_{\bar{b} b} & =\frac{3 g_{s}^{2} m_{b}^{2}\left(M^{2}-m_{b}^{2}\right)^{\frac{3}{2}}}{12 \pi M^{3}\left(M_{H}^{2}-4 M^{2}-i \Gamma_{H} M_{H}\right)^{2}}+\mathcal{O}\left(\left\langle v_{r}^{2}\right\rangle\right) .
\end{aligned}
$$

In this case, the leading contribution is given by the Higgs portal and the spin portal contributes to the next order in $v_{r}^{2}$. In Fig. 8, we show the predictions of the Higgs portal for the $\tau^{+} \tau^{-}$and $\bar{b} b$ channels. Clearly, these results are consistent with the upper bounds obtained by FermiLATDES. The spin portal yields even smaller values for the average cross section and it is also consistent with these upper bounds.

\section{DIRECT DETECTION OF DARK MATTER}

The large dark matter flux on Earth inferred from the measured dark matter density in our galaxy opened the possibility of direct detection of dark matter from its scattering by nuclei [46] and a lot of experimental effort has been done during the past few years using different techniques for the detection of the corresponding nuclei recoil kinetic energy $T$ (for a recent review see [47]).

The rate of interactions (counts per day per kilogram the nuclear kinetic energy recoil range $d T$ ) of a DM particle of mass $M$ with a nucleus of mass $M_{A}$ in the detector is given by

$$
\frac{d R}{d T}=\frac{\rho}{M M_{A}} \int|\mathbf{v}| f(\mathbf{v}) \frac{d \sigma}{d T}(T, \mathbf{v}) d^{3} \mathbf{v},
$$

where $\rho$ stands for the local dark matter number density, $\frac{d \sigma}{d T}(T, \mathbf{v})$ is the dark matter-nucleus differential cross section, $f(\mathbf{v})$ denotes the local distribution of dark matter velocities on Earth. The integral goes from $v_{\min }(T)$, the minimal velocity of an incoming dark matter to produce a nuclear recoil energy $T$ to $v_{\text {esc }}=557 \mathrm{~km} / \mathrm{sec}$, the escape velocity for a dark matter particle in our galaxy (the maximum velocity a dark matter particle bounded in the halo of our galaxy can have).

The rate of interactions is measured in the laboratory (LAB) system, thus we will calculate all the involved quantities in this reference frame. A straightforward calculation of the differential cross section for the $D\left(p_{1}\right) N_{A}\left(p_{2}\right) \rightarrow D\left(p_{3}\right) N_{A}\left(p_{4}\right)$ in the LAB system where $p_{1}=\left(E_{1}, \mathbf{p}_{1}\right), p_{2}=\left(M_{A}, \mathbf{0}\right), p_{3}=\left(E_{3}, \mathbf{p}_{3}\right), p_{4}=\left(M_{A}+\right.$ $T, \mathbf{p}_{A}$ ) yields

$$
\frac{d \sigma}{d T}(T, \mathbf{v})=\frac{|\overline{\mathcal{M}}(s, t, u)|^{2}}{32 \pi M_{A} \mathbf{p}_{1}^{2}} .
$$

The Mandelstam variables in the LAB frame are given by

$$
s=\left(E_{1}+M_{A}\right)^{2}-\mathbf{p}_{1}^{2}=\left(M+M_{A}\right)^{2}+M M_{A} \mathbf{v}^{2}+\mathcal{O}\left(\mathbf{v}^{4}\right),
$$

$$
\begin{gathered}
t=T^{2}-\left|\mathbf{p}_{A}\right|^{2}=-2 M_{A} T, \\
u=2 M^{2}+2 M_{A}^{2}-s-t=\left(M-M_{A}\right)^{2}+2 M_{A} T \\
-M M_{A} \mathbf{v}^{2}+\mathcal{O}\left(\mathbf{v}^{4}\right),
\end{gathered}
$$

where we wrote the incoming momentum as $\mathbf{p}_{1}=M \mathbf{v}$. Notice that the average squared amplitude depends only on the nuclear recoil energy and the incoming dark matter velocity. For a given incoming momentum $p_{1}$, the nuclear recoil energy is given by

$T=\frac{2 M_{A} M^{2} \mathbf{v}^{2} \cos ^{2} \theta}{\left(E_{1}+M_{A}\right)^{2}-M^{2} \mathbf{v}^{2} \cos ^{2} \theta}=\frac{2 M_{A} M^{2} \mathbf{v}^{2} \cos ^{2} \theta}{\left(M+M_{A}\right)^{2}}+\mathcal{O}\left(\mathbf{v}^{4}\right)$,

where $\theta$ stands for the nuclear recoil angle measured with respect to the direction of the incoming dark matter particle. The minimal velocity required to produce an energy recoil $T$ is obtained when the DM particle transfers the maximum momentum to the nucleus $(\theta=0)$ and it is given by

$$
v_{\min }^{2}(T)=\frac{\left(M+M_{A}\right)^{2}}{2 M_{A} M^{2}} T=\frac{M_{A}}{2 \mu_{A}^{2}} T,
$$

where $\mu_{A}$ stands for the dark matter-nucleus reduced mass.

The calculation of the invariant amplitude $\mathcal{M}$ requires to work out the effective theory for the nucleus dark matter interactions. The starting point is the fundamental theory for the interactions of dark mater with standard model particles. Then, an effective theory for the interactions of dark matter with nucleons can be built, and finally, the effective theory for interactions with nuclei is constructed. An important point is that for low momentum transfer processes, the finite size of the nucleus must be incorporated. At the end, taking into account the finite size of the nucleus, the amplitude can be written as

$$
\mathcal{M}=\mathcal{M}_{0} F_{S I}\left(q^{2}\right)
$$

where $\mathcal{M}_{0}$ is calculated with the effective theory at the nuclear level and $F_{S I}\left(q^{2}\right)$ is the nucleus form factor, in such a way that

$$
\frac{d \sigma}{d T}(T, \mathbf{v})=\frac{\left|\overline{\mathcal{M}}_{0}(s, t, u)\right|^{2}}{32 \pi M_{A} M^{2} \mathbf{v}^{2}} F_{S I}^{2}(t)
$$

In general, the differential cross section is given by Eq. (53). However, experimental results are reported in 
terms of the total $D-N_{A}$ cross section at zero momentum transfer. This comes about because the $D-N_{A}$ scattering takes place at low momentum transfer, which in the LAB frame means low $T$ and the total cross section at zero momentum transfer is a good starting point for the description of the process. Indeed, for example, in the XENON1T experiment, the detector is sensitive to $T \in[3,50] \mathrm{KeV}$. On the other side, the velocity of DM particles on Earth are in the range $|\mathbf{v}| \in\left[0, v_{\text {esc }}\right]$ with $v_{\text {esc }} / c=1.85 \times 10^{-3}$ and an average velocity $v_{0} / c=$ $0.73 \times 10^{-3}$.

It is conventionally assumed also that the interactions of standard model particles with dark matter are exchanged by a massive particle. In this case, in the effective theory, the leading term is a four-point interaction with an effective coupling suppressed as the mass of the exchanged particle and the same happens at the nuclear level. Said in other words, the average squared amplitude $\left|\overline{\mathcal{M}}_{0}(s, t, u)\right|^{2} \equiv$ $g\left(T, \mathbf{v}^{2}\right)$ is a regular function of $T$ and can be expanded as

$$
g\left(T, \mathbf{v}^{2}\right)=g_{0}\left(\mathbf{v}^{2}\right)+g_{1}\left(\mathbf{v}^{2}\right) T+\cdots
$$

and since the process takes place at low $T$, we can keep only the leading term in this expansion and the leading term in the expansion of the form factor. In this simplified scenario,

$$
\frac{d \sigma}{d T}(T, \mathbf{v}) \approx \frac{g_{0}\left(\mathbf{v}^{2}\right)}{32 \pi M_{A} M^{2} \mathbf{v}^{2}} F^{2}(0) .
$$

Notice that strictly speaking this is the differential cross section at zero momentum transfer. This quantity can be related to the total cross section integrating on $T$ (this is not required by the experiment but it is convenient for comparison purposes of the different experimental set up) from 0 to $T_{\max }=2 \mu_{A}^{2} \mathbf{v}^{2} / M_{A}$. We obtain

$$
\sigma(\mathbf{v}) \approx \frac{g_{0}\left(\mathbf{v}^{2}\right)}{32 \pi M_{A} M^{2} \mathbf{v}^{2}} \frac{2 \mu_{A}^{2} \mathbf{v}^{2}}{M_{A}} \approx \frac{\mu_{A}^{2} g_{0}(0)}{16 \pi M_{A}^{2} M^{2}} \equiv \sigma_{S I},
$$

where in the last step we expanded also in $g_{0}\left(\mathbf{v}^{2}\right)$ around $\mathbf{v}^{2}=0$ and kept only the leading term. In terms of the static quantity $\sigma_{S I}$, we can write the actual differential cross section in Eq. (53) to leading order in $T$ (in the dynamics) as

$$
\frac{d \sigma}{d T}(T, \mathbf{v})=\frac{M_{A}}{2 \mu_{A}^{2} \mathbf{v}^{2}} \sigma_{S I} F_{S I}^{2}(T),
$$

where $\sigma_{S I}$ is the $D-N_{A}$ total cross section at zero momentum transfer. This framework, which up to this point takes into account only the point interactions of dark matter with the nucleus, is then generalized to consider the possibility of spin-dependent interactions (motivated mainly by the axial coupling of the neutralino, see [48]) and the differential cross section is parametrized as

$$
\frac{d \sigma}{d T}(T, \mathbf{v})=\frac{M_{A}}{2 \mu_{A}^{2} \mathbf{v}^{2}}\left[\sigma_{S I} F_{S I}^{2}(T)+\sigma_{S D} F_{S D}^{2}(T)\right],
$$

where the possibility of a different response function for the spin-dependent interactions is considered through the corresponding form factor $F_{S D}^{2}(T)$. In our case, we have dominant spin-independent interactions, and we will focus on them in the following.

The most recent data on direct dark matter detection are given by XENON1T [49], who assume isospin conserving dark matter-nucleus interactions and report the following observable [50]

$$
\sigma_{p}=\frac{\mu_{p}^{2}}{A^{2} \mu_{A}^{2}} \sigma_{S I},
$$

where $\mu_{p}$ stands for the dark matter-proton reduced mass. In our formalism, dark matter-nucleus interactions are mediated by $H, Z^{0}$ and $\gamma$ and are not isospin conserving. Furthermore, the exchange of massive particles like $H$ and $Z$ produce four-point dark matter-nucleus interactions for small momentum transfer and follows the treatment reviewed above, but the exchange of photons, whose propagator has a pole at $q^{2}=0$, departs from this picture and we must modify the above relations accordingly in order to calculate properly the observable reported by XENON1T, which does not correspond with the zeromomentum dark matter-proton cross section if interactions are not isospin invariant. For this purpose, we rewrite the $D-N_{A}$ differential cross section in Eq. (53) as

$$
\frac{d \sigma}{d T}(T, \mathbf{v})=\frac{\xi}{\mathbf{v}^{2}} g\left(T, \mathbf{v}^{2}\right) F_{S I}^{2}(T),
$$

where $\xi=\left(32 \pi M_{A} M^{2}\right)^{-1}$ but now $g\left(T, \mathbf{v}^{2}\right)$ is not a regular function of $T$ due to the poles of the exchanged massless particle. In this case, it is not possible to perform an expansion around $T=0$, but experiments start detecting nuclear recoil at a given $T=T_{\min }$ anyway and we can expand around this value of $T$

$$
\begin{aligned}
\frac{d \sigma}{d T}(T, \mathbf{v})= & \frac{\xi}{\mathbf{v}^{2}}\left[g\left(T_{\min }, \mathbf{v}^{2}\right)+g^{\prime}\left(T_{\min }, \mathbf{v}^{2}\right)\left(T-T_{\min }\right)\right] \\
& \times\left[F^{2}\left(T_{\min }\right)+\left(F^{2}\right)^{\prime}\left(T_{\min }\right)\left(T-T_{\min }\right)\right] \\
= & \frac{\xi}{\mathbf{v}^{2}} g\left(T_{\min }, \mathbf{v}^{2}\right) F_{S I}^{2}\left(T_{\min }\right)+\mathcal{O}\left(T-T_{\min }\right) .
\end{aligned}
$$

Integrating now from $T_{\min }$ to $T_{\max }=2 \mu_{A}^{2} \mathbf{v}^{2} / M_{A}$ and keeping only the leading term, we get

$$
\sigma_{A}=\frac{\xi}{\mathbf{v}^{2}} g\left(T_{\min }, \mathbf{v}^{2}\right) F_{S I}^{2}\left(T_{\min }\right)\left(T_{\max }-T_{\min }\right) .
$$

This is the total dark matter-nucleus cross section at the fixed momentum transfer $q^{2}=-2 M_{A} T_{\min }$. The differential 
cross section can be rewritten in terms of this static quantity and takes the form in Eq. (57) but now

$$
\sigma_{S I}=\frac{\sigma_{A}}{F_{S I}^{2}\left(T_{\min }\right)} \frac{T_{\max }}{T_{\max }-T_{\min }}=\frac{\mu_{A}^{2}}{16 \pi M_{A}^{2} M^{2}} g\left(T_{\min }, \mathbf{v}^{2}\right) .
$$

As it will be shown below, in our formalism the average squared amplitude has the following form

$$
g\left(T, \mathbf{v}^{2}\right)=a_{0}+\left(\frac{b_{0}}{T}+c_{0}\right) \mathbf{v}^{2}+\mathcal{O}\left(T, \mathbf{v}^{4}\right)
$$

The observable $\sigma_{p}$ reported by XENON is then given by $\sigma_{p}=\frac{1}{16 \pi A^{4}\left(M+M_{p}\right)^{2}}\left[a_{0}+\left(\frac{b_{0}}{T_{\min }}+c_{0}\right) \mathbf{v}^{2}+\mathcal{O}\left(T, \mathbf{v}^{4}\right)\right]$.

In principle, the leading contribution is given by $a_{0}$ since $\mathbf{v}^{2}$ is small. However, the photon contribution produces the $\mathcal{O}\left(\mathbf{v}^{2}\right)$ term $b_{0} \mathbf{v}^{2} / T$, which is enhanced by the photon pole and could give sizable contributions, thus we keep this term in the calculations. In comparison, the contributions from the $c_{0}$ term are clearly small and we neglect it in the following. Under these considerations we get

$$
\sigma_{p}=\frac{1}{16 \pi A^{4}\left(M+M_{p}\right)^{2}}\left[a_{0}+\frac{b_{0} \mathbf{v}^{2}}{T_{\min }}\right] .
$$

The calculation of the $D-N_{A}$ differential cross section requires to work out the effective interactions of $H, \gamma$ and $Z^{0}$ with nuclei, which in turn requires to work out first the effective theory for the interactions with nucleons. At the nucleon level, the corresponding effective Lagrangian is given by [51]

$$
\begin{aligned}
\mathcal{L}_{\text {eff }}^{N}= & \sum_{N=p, n}\left(g_{H N N} H \bar{N} N-e \bar{N} Q_{N} \gamma^{\mu} N A_{\mu}\right. \\
& \left.-\frac{M_{Z}}{2 v} \bar{N} \gamma^{\mu}\left(A_{N}+B_{N} \gamma^{5}\right) N Z_{\mu}\right),
\end{aligned}
$$

where $Q_{p}=1, Q_{n}=0$ and

$$
\begin{gathered}
g_{H N N}=-\left(7 \sum_{u, d, s} f_{T q}^{(N)}+2\right) \frac{m_{N}}{9 v}, \\
A_{p}=2 A_{u}+A_{d}=1-4 \sin ^{2} \theta_{W}, \\
A_{n}=A_{u}+2 A_{d}=-1,
\end{gathered}
$$

and

$$
\begin{gathered}
B_{N}=-\Delta_{u}^{(N)}+\Delta_{d}^{(N)}+\Delta_{s}^{(N)}, \\
B_{p}=-\Delta_{u}^{(p)}+\Delta_{d}^{(p)}+\Delta_{s}^{(p)}, \\
B_{n}=-\Delta_{d}^{(p)}+\Delta_{u}^{(p)}+\Delta_{s}^{(p)} .
\end{gathered}
$$

In our calculations, we use the specific values given in Table I.

At the nuclear level, the effective lagrangian has a similar form

$$
\begin{aligned}
\mathcal{L}_{\text {eff }}^{A}= & g_{H N_{A} N_{A}} H \bar{N}_{A} N_{A}-Z e \bar{N}_{A} \gamma^{\mu} N_{A} A_{\mu} \\
& -\frac{M_{Z}}{2 v} \bar{N}_{A} \gamma^{\mu}\left(A_{A}+B_{A} \gamma^{5}\right) N_{A} Z_{\mu},
\end{aligned}
$$

with

$$
\begin{aligned}
g_{H N_{A} N_{A}} & =Z g_{H p p}+(A-Z) g_{H n n}, \\
A_{A} & =Z A_{p}+(A-Z) A_{n}, \\
B_{A} & =Z B_{p}+(A-Z) B_{n},
\end{aligned}
$$

where $Z$ stands for the atomic number and $A$ denotes the total number of nucleons inside the nucleus.

The invariant amplitude for $D\left(p_{1}\right) N_{A}\left(p_{2}\right) \rightarrow$ $D\left(p_{3}\right) N_{A}\left(p_{4}\right)$ is induced by $t$-channel exchange of $H, \gamma$ and $Z^{0}$. The corresponding contributions are given by

$$
-i \mathcal{M}_{H}=i \frac{g_{D N_{A} H}}{t-m_{H}^{2}} \bar{U}\left(p_{3}\right)\left(g_{s} I+i g_{p} \chi\right) U\left(p_{1}\right) \bar{N}_{A}\left(p_{4}\right) N_{A}\left(p_{2}\right),
$$

$$
\begin{aligned}
-i \mathcal{M}_{\gamma}= & -\frac{g_{D N_{A} \gamma}}{t} \bar{U}\left(p_{3}\right) M_{\alpha \beta}\left(p_{1}-p_{3}\right)^{\beta} U\left(p_{1}\right) \bar{N}_{A} \\
& \times\left(p_{4}\right) \gamma^{\alpha} N_{A}\left(p_{2}\right), \\
-i \mathcal{M}_{Z}= & \frac{g_{D N_{A} Z}}{t-M_{Z}^{2}} \bar{U}\left(p_{3}\right) M_{\alpha \beta}\left(p_{1}-p_{3}\right)^{\beta} U\left(p_{1}\right) \bar{N}_{A} \\
& \times\left(p_{4}\right) \gamma^{\alpha}\left(A_{A}+B_{A} \gamma_{5}\right) N_{A}\left(p_{2}\right),
\end{aligned}
$$

where

$$
\begin{gathered}
g_{D N_{A} H}=-v g_{H N_{A} N_{A}}, \\
g_{D N_{A} \gamma}=2 Z e g_{t} \cos \theta_{W},
\end{gathered}
$$

TABLE I. Values for the coefficients $f_{T q}^{(p)}, f_{T q}^{(n)}$ and $\Delta_{q}^{(p)}$ extracted from Ref. [52].

\begin{tabular}{lllr}
\hline \hline & $f_{T q}^{(p)}$ & $f_{T q}^{(n)}$ & $\Delta_{q}^{(p)}$ \\
\hline$u$ & 0.023 & 0.019 & 0.77 \\
$d$ & 0.034 & 0.041 & -0.40 \\
$s$ & 0.14 & 0.14 & -0.12 \\
\hline \hline
\end{tabular}




$$
g_{D N_{A} Z}=\frac{M_{Z} g_{t} \sin \theta_{W}}{v}
$$

The leading contributions are spin-independent and come from the Higgs and photon exchange, and from the weak vector current in the $Z^{0}$ exchange. The leading spin-dependent terms come from the axial current. We perform a covariant calculation of the squared amplitude and identify the leading spin-independent contributions, spin-dependent terms are suppressed by powers of $\mathbf{v}^{2}$ in our formalism. The average squared amplitude is

$$
\begin{aligned}
|\overline{\mathcal{M}}|^{2}= & \frac{g_{D N_{A} H}^{2}}{6 M^{4}\left(t-m_{H}^{2}\right)^{2}}\left(4 M_{A}^{2}-t\right)\left(\left(g_{p}^{2}+g_{s}^{2}\right) t\left(t-4 M^{2}\right)+6 g_{s}^{2} M^{4}\right)+\frac{g_{D N_{A} \gamma}^{2}}{3 M^{4} t}\left[-2 M_{A}^{4}\left(M^{2}-t\right)+M_{A}^{2}\left(4 M^{4}+4 M^{2}(s+t)\right.\right. \\
& \left.-2 t(2 s+t))-2 M^{6}+2 M^{4}(2 s+3 t)-M^{2}\left(2 s^{2}+6 s t+t^{2}\right)+2 s t(s+t)\right] \\
& +\frac{g_{D N_{A} Z}^{2}}{3 M^{4}\left(t-M_{Z}^{2}\right)^{2}}\left[\left(A_{A}^{2}+B_{A}^{2}\right)\left(2 M_{A}^{4}\left(t-M^{2}\right)-2 M^{6}+2 M^{4}(2 s+3 t)-M^{2}\left(2 s^{2}+6 s t+t^{2}\right)+2 s t(s+t)\right)\right. \\
& \left.+M_{A}^{2}\left(A_{A}^{2}\left(4 M^{4}+4 M^{2}(s+t)-2 t(2 s+t)\right)-4 B_{A}^{2}\left(3 M^{4}-M^{2} s+s t\right)\right)\right] t \\
& -\left(\frac{g_{D N_{A} H} g_{D N_{A} \gamma}}{t-m_{H}^{2}}\right)\left(\frac{2 g_{s} M_{A}}{3 M^{4}}\right)\left(2 M^{2}-t\right)(s-u)+\frac{g_{D N_{A} H} g_{D N_{A} Z}}{\left(t-m_{H}^{2}\right)\left(t-M_{Z}^{2}\right)}\left(\frac{2 A_{A} g_{s} M_{A}}{3 M^{4}}\right)\left(2 M^{2}-t\right)(s-u) t \\
& -\frac{2 A_{A}}{3 M^{4}} \frac{g_{D N_{A} \gamma} g_{D N_{A} Z}}{\left(t-M_{Z}^{2}\right)}\left[-2 M_{A}^{4}\left(M^{2}-t\right)+M_{A}^{2}\left(4 M^{4}+4 M^{2}(s+t)-2 t(2 s+t)\right)-2 M^{6}+2 M^{4}(2 s+3 t)\right. \\
& \left.-M^{2}\left(2 s^{2}+6 s t+t^{2}\right)+2 s t(s+t)\right] .
\end{aligned}
$$

Expanding the average squared amplitude and keeping the leading terms in $\mathbf{v}^{2}$ and $T$ we get

$$
\begin{aligned}
|\overline{\mathcal{M}}|^{2}= & \frac{4 g_{s}^{2} g_{D N_{A} H}^{2} M_{A}^{2}}{m_{H}^{4}}+\frac{2 g_{D N_{A} \gamma}^{2}}{3 M^{2}}\left(M^{2}-2 M M_{A}+3 M_{A}^{2}\right)+\frac{16 g_{s} g_{D N_{A} \gamma} g_{D N_{A} H} M_{A}^{2}}{3 M m_{H}^{2}} \\
& +\left(\frac{4 g_{D N_{A} \gamma}^{2} M_{A}}{3 T}-\frac{16 A_{A} g_{D N_{A} \gamma} g_{D N_{A} Z} M_{A}^{2}}{3 M_{Z}^{2}}+\frac{8 g_{s} g_{D N_{A} \gamma} g_{D N_{A} H} M_{A}^{2}}{3 M m_{H}^{2}}-\frac{2 g_{D N_{A} \gamma}^{2} M_{A}}{3 M^{2}}\left(M-4 M_{A}\right)\right) \mathbf{v}^{2} .
\end{aligned}
$$

Notice that all terms in this equation contribute to the spin-independent cross section. Leading contributions to the spindependent cross section starts at order $\mathbf{v}^{4}$. This is a consequence of the coupling of the $Z^{0}$ to the higher multipoles of the dark matter instead of the weak charges (they are standard model singlets). Here, we identify the coefficients in Eq. (64) as

$$
\begin{gathered}
a_{0}=\frac{4 g_{s}^{2} g_{D N_{A} H}^{2} M_{A}^{2}}{m_{H}^{4}}+\frac{2 g_{D N_{A} \gamma}^{2}}{3 M^{2}}\left(M^{2}-2 M M_{A}+3 M_{A}^{2}\right)+\frac{16 g_{s} g_{D N_{A} \gamma} g_{D N_{A} H} M_{A}^{2}}{3 M m_{H}^{2}}, \\
b_{0}=\frac{4 g_{D N_{A} \gamma}^{2} M_{A}}{3}, \\
c_{0}=-\frac{16 A_{A} g_{D N_{A} \gamma} g_{D N_{A} Z} M_{A}^{2}}{3 M_{Z}^{2}}+\frac{8 g_{s} g_{D N_{A} \gamma} g_{D N_{A} H} M_{A}^{2}}{3 M m_{H}^{2}}-\frac{2 g_{D N_{A} \gamma}^{2} M_{A}}{3 M^{2}}\left(M-4 M_{A}\right) .
\end{gathered}
$$

In Fig. 9, we show our results for the observable $\sigma_{p}$ in Eq. (66) as a function of the dark matter mass $M$ for different values of the couplings $g_{t}$ and $g_{s}$. In these plots, we consider $A=131, Z=54$ and $T_{\min }=3 \mathrm{KeV}$ as appropriate for XENON experiment and compare with the recently published XENON1T results [49]. For the spin portal $\left(g_{s}=0\right)$ the leading contributions are due to the photon exchange and are dominated by the photon pole. From these plots we conclude that dark matter with a $(1,0) \oplus(0,1)$ spacetime structure is consistent with XENON1T measurements of $\sigma_{p}$, for both spin and Higgs portals, although the spin portal coupling must be small $\left(g_{t} \approx 10^{-4}\right)$ if the dark matter mass is of the order of a few hundreds $\mathrm{GeV}$. 

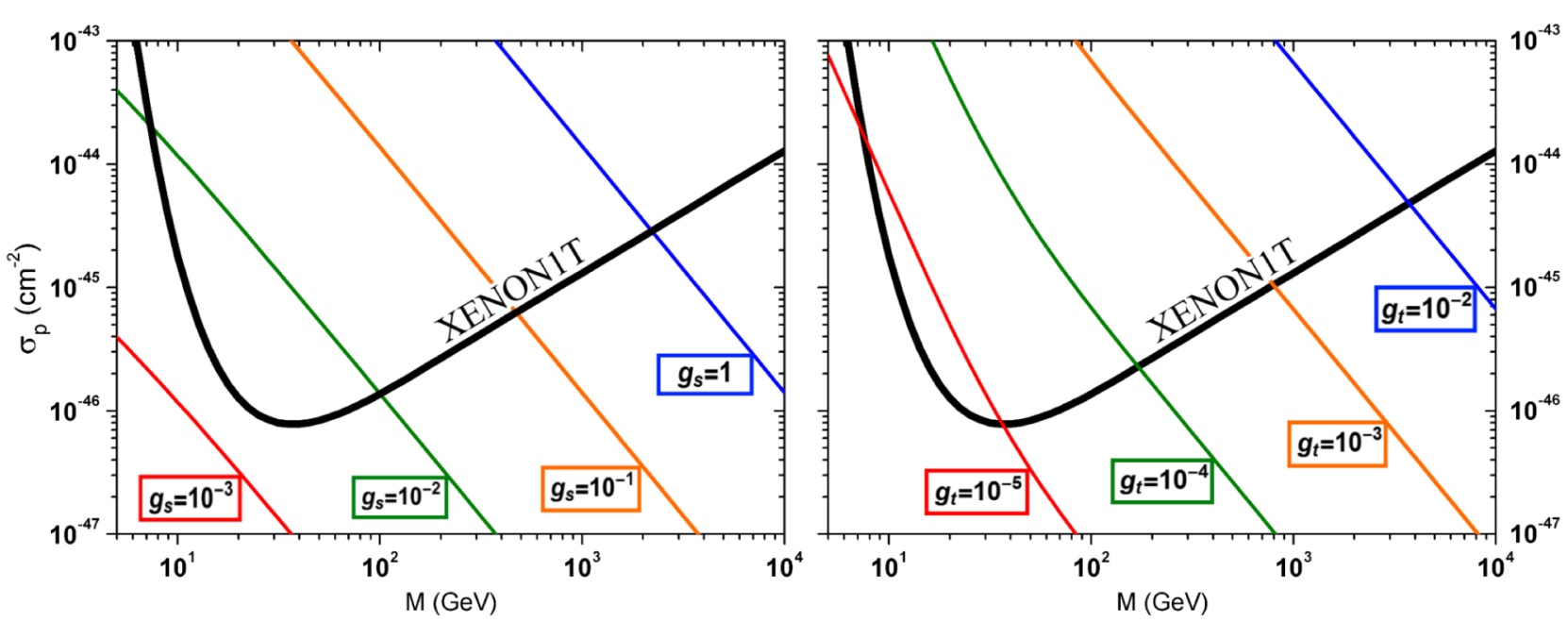

FIG. 9. Observable $\sigma_{p}$ as a function of the dark matter mass for the Higgs $\left(g_{t}=0\right.$, left panel) and spin $\left(g_{s}=0\right.$, right panel $)$ portals, compared with the XENON1T upper bounds [49].

\section{CONCLUSIONS}

Effective theories for the interaction of dark matter with standard model particles has been done mainly assuming spacetime structures for dark matter similar to those of the standard model fields, i.e., dark matter fields transforming in the $(0,0),\left(\frac{1}{2}, 0\right) \oplus\left(0, \frac{1}{2}\right)$ or $\left(\frac{1}{2}, \frac{1}{2}\right)$ representations of the homogeneous Lorentz group.

In this work, we study the possibility of a $(1,0) \oplus(0,1)$ spacetime structure for dark matter fields. Assuming that dark matter fields are standard model singlets, we find three lowest order terms which are dimension four in the corresponding effective theory. Two of them couple the Higgs to dark matter yielding a Higgs portal and the third one couples the photon and $Z^{0}$ fields to higher multipoles of the spin-one dark matter fields, yielding a spin portal to dark matter.

We study the phenomenology derived from our proposal starting with the possibility of light dark matter $\left(M<M_{Z} / 2\right.$ or $\left.M_{H} / 2\right)$, in which case the $H \rightarrow \bar{D} D$ or $Z^{0} \rightarrow \bar{D} D$ are kinematically permitted and contribute to the Higgs and $Z^{0}$ invisible decay widths. We use experimental results on these widths to put upper limits to the corresponding low energy constants. In general, for light dark matter mass we find stringent constraints for the couplings of the Higgs portal: $g_{s}, g_{p} \leq 10^{-3}$ and less stringent constraints on the spin portal coupling $g_{t}$.

For dark matter mass in this region, nonrelativistic dark matter can annihilate into a photon pair or into a fermionantifermion pair if $M>m_{f}$. We calculate these processes in our formalism and use them to calculate the corresponding dark matter relic density for both the Higgs and the spin portal. For the spin portal, taking into account the constraints from the $Z^{0}$ invisible width, we find that a proper description of the measured dark matter relic density imposes the lower bound $M>43 \mathrm{GeV}$ for dark matter with a $(1,0) \oplus(0,1)$ spacetime structure. For the Higgs portal, similar considerations yields the lower bound $M>62 \mathrm{GeV}$.

For higher dark matter mass $\left(M>M_{H} / 2\right)$, the $Z^{0} \rightarrow$ $\bar{D} D$ and $H \rightarrow \bar{D} D$ decays are kinematically forbidden and we lose the corresponding constraints on the low energy couplings $g_{t}, g_{s}, g_{p}$. In order to test our proposal in this energy region, we calculate the velocity averaged cross section for the annihilation of dark matter into $\tau^{+} \tau^{-}$and $\bar{b} b$ in Milky Way dwarf spheroid satellite galaxies and compare with recent upper bounds from a joint analysis by DES and FermiLAT Collaborations, finding that both portals yield results consistent with these upper bounds.

Another very active source of experimental information is given by direct detection experiments. In this case, it is usually assumed that dark matter-nucleus interaction is described by a four-point interaction, which is appropriate when this interaction is exchanged by a heavy particle. We elaborate on this picture to account for the exchange of photons (whose contributions are suppressed anyway because it couples to the higher multipoles of the dark matter) and calculate the observable $\sigma_{p}$ reported recently by the XENON Collaboration. The Higgs portal yields results consistent with the upper bounds reported by XENON1T. In the case of the spin portal, consistency with XENON1T results requires small values of the spin portal coupling or large values of the dark matter mass.

\section{ACKNOWLEDGMENTS}

Work supported by CONACyT México under Project No. CB-259228. H. H. A. acknowledges CONACyT for a scholarship and DAIP-UG for a grant under the Call for Support to Graduate Studies 2017. 
APPENDIX: TRACEOLOGY FOR $(1,0) \oplus(0,1)$

In this Appendix, we collect the trace relations necessary for the calculations in this work. The covariant basis for the $(1,0) \oplus(0,1)$ representation space is given by the set of $6 \times 6$ matrices $\left\{\mathbf{1}, \chi, S^{\mu \nu}, \chi S^{\mu \nu}, M^{\mu \nu}, C^{\mu \nu \alpha \beta}\right\}$ where 1 is the identity matrix. The first principles construction of these matrices can be found in [38] and their explicit form depends on the basis chosen for the states in the $(1,0) \oplus$ $(0,1)$ representation. All the calculations in this work are representation independent and rely only on their algebraic properties. The starting point are first principles construction of the rest-frame parity operator $(\Pi)$, the Lorentz generators $J^{i}=\frac{1}{2} \epsilon^{i j k} M^{j k}$ and $K^{i}=M^{0 i}$ and the chirality operator $\chi$ entering the projectors on the chiral subspaces $(1,0)$ and $(0,1)$ which satisfy

$$
\{\chi, \Pi\}=0, \quad\left[\chi, M^{\mu \nu}\right]=0, \quad \chi^{2}=\mathbf{1} .
$$

The $S^{\mu \nu}$ tensor is the covariant version of the rest-frame parity operator ( $\Pi$ ) such that $S^{00}=\Pi$ and other components can be written as

$$
S^{\mu \nu}=\Pi\left(g^{\mu \nu}-i\left(g^{0 \mu} M^{0 \nu}+g^{0 \nu} M^{0 \mu}\right)-\left\{M^{0 \mu}, M^{0 \nu}\right\}\right) .
$$

This is a symmetric traceless $\left(S_{\mu}^{\mu}=0\right)$ tensor with nine independent components. As a consequence of Eqs. (A1), we get

$$
\left\{\chi, S^{\mu \nu}\right\}=0 .
$$

The $C$ tensor is given by

$$
\begin{aligned}
C^{\mu \nu \alpha \beta}= & 4\left\{M^{\mu \nu}, M^{\alpha \beta}\right\}+2\left\{M^{\mu \alpha}, M^{\nu \beta}\right\}-2\left\{M^{\mu \beta}, M^{\nu \alpha}\right\} \\
& -8\left(g^{\mu \alpha} g^{\nu \beta}-g^{\mu \beta} g^{\nu \alpha}\right),
\end{aligned}
$$

with the symmetry properties $C^{\mu \nu \alpha \beta}=-C^{\nu \mu \alpha \beta}=-C^{\mu \nu \beta \alpha \text {; }}$ $C^{\mu \nu \alpha \beta}=C^{\alpha \beta \mu \nu}$. It satisfies the Bianchi identity $C^{\mu \alpha \beta \nu}+$ $C^{\mu \beta \nu \alpha}+C^{\mu \nu \alpha \beta}=0$ and the contraction of any pair of indices vanishes $C_{\nu}^{\nu \alpha \beta}=0$. These constraints leave only 10 independent components. Clearly it satisfies $\left[\chi, C^{\mu \nu \alpha \beta}\right]=0$.

The covariant basis is orthogonal with respect to the scalar product defined as $\langle A \mid B\rangle=\operatorname{Tr}(A B)$, thus these matrices satisfy the following relations

$$
\begin{aligned}
\operatorname{Tr}(\chi) & =\operatorname{Tr}(S)=\operatorname{Tr}(M)=\operatorname{Tr}(\chi S)=\operatorname{Tr}(C)=0, \\
\operatorname{Tr}(\chi M) & =\operatorname{Tr}(\chi C)=\operatorname{Tr}(M S)=\operatorname{Tr}(M \chi S) \\
& =\operatorname{Tr}(M C)=\operatorname{Tr}(S \chi S)=\operatorname{Tr}(S C)=\operatorname{Tr}(\chi S C)=0,
\end{aligned}
$$

where we suppressed the Lorentz indices.
Calculations in this work requires traces of products of the $S^{\mu \nu}$ tensor and other elements in the covariant basis. Let us consider first

$$
\begin{aligned}
\operatorname{Tr}(S M M) & =\operatorname{Tr}\left(\chi^{2} S M M\right)=-\operatorname{Tr}(\chi S \chi M M) \\
& =-\operatorname{Tr}(\chi S M M \chi)=-\operatorname{Tr}(S M M) \\
& \Rightarrow \operatorname{Tr}(S M M)=0,
\end{aligned}
$$

where we used Eqs. (A1), (A3) and the cyclic property of a trace. Since $\chi$ commutes also with $C$, this procedure can be used to show that in general if we have a term with an odd numbers of $S$ tensors the trace of this term will vanish

$$
\operatorname{Tr}\left(\text { term with an odd of } S^{\prime} \mathrm{s}\right)=0 .
$$

The trace of terms with an even number of $S$ factors can always be reduced to a linear combination of terms with the trace of the product of two $S$ or two $M$ factors using the following (anti)commutation relations

$$
\left[M^{\mu \nu}, M^{\alpha \beta}\right]=-i\left(g^{\mu \alpha} M^{\nu \beta}-g^{\nu \alpha} M^{\mu \beta}-g^{\mu \beta} M^{\nu \alpha}+g^{\nu \beta} M^{\mu \alpha}\right),
$$

$$
\left\{M^{\mu \nu}, M^{\alpha \beta}\right\}=\frac{4}{3}\left(g^{\mu \alpha} g^{\nu \beta}-g^{\mu \beta} g^{\nu \alpha}\right)-\frac{4}{3} i \varepsilon^{\mu \nu \alpha \beta} \chi+\frac{1}{6} C^{\mu \nu \alpha \beta},
$$

$$
\left[M^{\mu \nu}, S^{\alpha \beta}\right]=-i\left(g^{\mu \alpha} S^{\nu \beta}-g^{\nu \alpha} S^{\mu \beta}+g^{\mu \beta} S^{\nu \alpha}-g^{\nu \beta} S^{\mu \alpha}\right),
$$

$$
\left\{M^{\mu \nu}, S^{\alpha \beta}\right\}=\varepsilon^{\mu \nu \sigma \beta} \chi S_{\sigma}^{\alpha}+\varepsilon^{\mu \nu \sigma \alpha} \chi S_{\sigma}^{\beta},
$$

$$
\left[S^{\mu \nu}, S^{\alpha \beta}\right]=-i\left(g^{\mu \alpha} M^{\nu \beta}+g^{\nu \alpha} M^{\mu \beta}+g^{\nu \beta} M^{\mu \alpha}+g^{\mu \beta} M^{\nu \alpha}\right),
$$

$$
\begin{aligned}
\left\{S^{\mu \nu}, S^{\alpha \beta}\right\}= & \frac{4}{3}\left(g^{\mu \alpha} g^{\nu \beta}+g^{\nu \alpha} g^{\mu \beta}-\frac{1}{2} g^{\mu \nu} g^{\alpha \beta}\right) \\
& -\frac{1}{6}\left(C^{\mu \alpha \nu \beta}+C^{\mu \beta \nu \alpha}\right) .
\end{aligned}
$$

The simplest case appears in the calculation of $H \rightarrow \bar{D} D$

$$
\begin{aligned}
\operatorname{Tr}\left(S^{\mu \nu} S^{\alpha \beta}\right) & =\operatorname{Tr}\left(\frac{1}{2}\left[S^{\mu \nu}, S^{\alpha \beta}\right]+\frac{1}{2}\left\{S^{\mu \nu}, S^{\alpha \beta}\right\}\right) \\
& =4\left(g^{\mu \alpha} g^{\nu \beta}+g^{\mu \beta} g^{\nu \alpha}-\frac{1}{2} g^{\mu \nu} g^{\alpha \beta}\right) \\
& \equiv 4 T^{\mu \nu \alpha \beta} .
\end{aligned}
$$

Similarly, the calculation of $Z^{0} \rightarrow \bar{D} D$ requieres 


$$
\operatorname{Tr}\left(M^{\mu \nu} M^{\alpha \beta}\right)=\operatorname{Tr}\left(\frac{1}{2}\left[M^{\mu \nu}, M^{\alpha \beta}\right]+\frac{1}{2}\left\{M^{\mu \nu}, M^{\alpha \beta}\right\}\right)=4\left(g^{\mu \alpha} g^{\nu \beta}-g^{\mu \beta} g^{\nu \alpha}\right) \equiv 4 G^{\mu \nu \alpha \beta}
$$

The first example of the reduction mentioned above is faced in the calculation of $Z^{0} \rightarrow \bar{D} D$ which also requires to calculate

$$
\begin{aligned}
\operatorname{Tr}\left(S^{\mu \nu} S^{\alpha \beta} M^{\rho \sigma}\right) & =\operatorname{Tr}\left(\frac{1}{2}\left\{S^{\mu \nu}, S^{\alpha \beta}\right\} M^{\rho \sigma}+\frac{1}{2}\left[S^{\mu \nu}, S^{\alpha \beta}\right] M^{\rho \sigma}\right) \\
& =\frac{-i}{2} \operatorname{Tr}\left(\left(g^{\mu \alpha} M^{\nu \beta}+g^{\nu \alpha} M^{\mu \beta}+g^{\nu \beta} M^{\mu \alpha}+g^{\mu \beta} M^{\nu \alpha}\right) M^{\rho \sigma}\right) \\
& =-2 i\left(g^{\mu \alpha} G^{\nu \beta \rho \sigma}+g^{\nu \alpha} G^{\mu \beta \rho \sigma}+g^{\nu \beta} G^{\mu \alpha \rho \sigma}+g^{\mu \beta} G^{\nu \alpha \rho \sigma}\right),
\end{aligned}
$$

and

$$
\begin{aligned}
& \operatorname{Tr}\left(S^{\alpha \beta} M^{\mu \nu} S^{\rho \sigma} M^{\gamma \delta}\right)=\operatorname{Tr}\left(\left(\frac{1}{2}\left[S^{\alpha \beta}, M^{\mu \nu}\right]+\frac{1}{2}\left\{S^{\alpha \beta}, M^{\mu \nu}\right\}\right)\left(\frac{1}{2}\left[S^{\rho \sigma}, M^{\gamma \delta}\right]+\frac{1}{2}\left\{S^{\rho \sigma}, M^{\gamma \delta}\right\}\right)\right) \\
& =\operatorname{Tr}\left(\left(\frac{i}{2}\left(g^{\mu \alpha} S^{\nu \beta}-g^{\nu \alpha} S^{\mu \beta}+g^{\mu \beta} S^{\nu \alpha}-g^{\nu \beta} S^{\mu \alpha}\right)-\varepsilon^{\mu \nu \tau \beta} \chi S_{\tau}^{\alpha}-\varepsilon^{\mu \nu \tau \alpha} \chi S_{\tau}^{\beta}\right)\right. \\
& \left.\times\left(\frac{i}{2}\left(g^{\gamma \rho} S^{\delta \sigma}-g^{\delta \rho} S^{\gamma \sigma}+g^{\gamma \sigma} S^{\delta \rho}-g^{\delta \sigma} S^{\gamma \rho}\right)-\varepsilon^{\gamma \delta \lambda \sigma} \chi S_{\lambda}^{\rho}-\varepsilon^{\gamma \delta \lambda \rho} \chi S_{\lambda}^{\sigma}\right)\right) \\
& =-g^{\mu \alpha} g^{\gamma \rho} T^{\nu \beta \delta \sigma}+g^{\mu \alpha} g^{\delta \rho} T^{\nu \beta \gamma \sigma}-g^{\mu \alpha} g^{\gamma \sigma} T^{\nu \beta \delta \rho}+g^{\mu \alpha} g^{\delta \sigma} T^{\nu \beta \gamma \rho}+g^{\nu \alpha} g^{\gamma \rho} T^{\mu \beta \delta \sigma}-g^{\nu \alpha} g^{\delta \rho} T^{\mu \beta \gamma \sigma}+g^{\nu \alpha} g^{\gamma \sigma} T^{\mu \beta \delta \rho} \\
& -g^{\nu \alpha} g^{\delta \sigma} T^{\mu \beta \gamma \rho}-g^{\mu \beta} g^{\gamma \rho} T^{\nu \alpha \delta \sigma}+g^{\mu \beta} g^{\delta \rho} T^{\nu \alpha \gamma \sigma}-g^{\mu \beta} g^{\gamma \sigma} T^{\nu \alpha \delta \rho}+g^{\mu \beta} g^{\delta \sigma} T^{\nu \alpha \gamma \rho}+g^{\nu \beta} g^{\gamma \rho} T^{\mu \alpha \delta \sigma}-g^{\nu \beta} g^{\delta \rho} T^{\mu \alpha \gamma \sigma}
\end{aligned}
$$

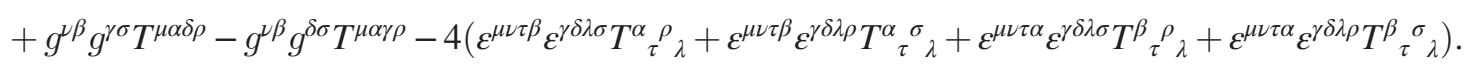

Similarly it can be shown that

$$
\begin{aligned}
\operatorname{Tr}\left(M^{\mu \nu} M^{\alpha \beta} M^{\rho \sigma}\right)= & -2 i\left(g^{\mu \alpha} G^{\nu \beta \rho \sigma}-g^{\nu \alpha} G^{\mu \beta \rho \sigma}\right. \\
& \left.-g^{\mu \beta} G^{\nu \alpha \rho \sigma}+g^{\nu \beta} G^{\mu \alpha \rho \sigma}\right)
\end{aligned}
$$

$\operatorname{Tr}\left(\chi S^{\gamma \delta} S^{\alpha \beta} M^{\mu \nu}\right)=-2\left(\varepsilon^{\mu \nu \sigma \beta} T_{\sigma}^{\gamma \delta \alpha}+\varepsilon^{\mu \nu \sigma \alpha} T_{\sigma}^{\gamma \delta \beta}\right)$,

$$
\operatorname{Tr}\left(\chi M^{\mu \nu} M^{\alpha \beta}\right)=-4 i \varepsilon^{\mu \nu \alpha \beta} .
$$

The calculation of the trace of terms involving six or eight $S$ or $M$ factors (with an even number of $S$ factors) needed in this paper are reduced in a similar way.

There is a simpler way to obtain these results however, which is specially useful for terms with six or more factors. Since the result rests only on the algebraic properties in Eqs. (A8), (A9), (A10), (A11), (A12), (A13), we can use any representation of these operators for the calculation of the trace. In this concern, the use of the representation where the internal matrix indices transform as Lorentz indices is convenient, since in this case the calculation of the trace reduces to contractions of Lorentz indices which can be easily done using conventional algebraic manipulation codes like FEYNCALC. In this representation, each internal matrix index $a$ is replaced by a pair of antisymmetric Lorentz indices $\alpha \beta$ [39]. The explicit form of the operators in the covariant basis is given by

$$
\begin{gathered}
(\mathbf{1})_{\alpha \beta \gamma \delta}=\frac{1}{2}\left(g_{\alpha \gamma} g_{\beta \delta}-g_{\alpha \delta} g_{\beta \gamma}\right), \\
(\chi)_{\alpha \beta \gamma \delta}=\frac{i}{2} \varepsilon_{\alpha \beta \gamma \delta}, \\
\left(M_{\mu \nu}\right)_{\alpha \beta \gamma \delta}=-i\left(g_{\mu \gamma} \mathbf{1}_{\alpha \beta \nu \delta}+g_{\mu \delta} \mathbf{1}_{\alpha \beta \gamma \nu}-g_{\gamma \nu} \mathbf{1}_{\alpha \beta \mu \delta}-g_{\delta \nu} \mathbf{1}_{\alpha \beta \gamma \mu}\right), \\
\left(S_{\mu \nu}\right)_{\alpha \beta \gamma \delta}=g_{\mu \nu} \mathbf{1}_{\alpha \beta \gamma \delta}-g_{\mu \gamma} \mathbf{1}_{\alpha \beta \nu \delta}-g_{\mu \delta} \mathbf{1}_{\alpha \beta \gamma \nu}-g_{\gamma \nu} \mathbf{1}_{\alpha \beta \mu \delta} \\
-g_{\delta \nu} \mathbf{1}_{\alpha \beta \gamma \mu} .
\end{gathered}
$$

The explicit form of $C^{\mu \nu \alpha \beta}$ can be constructed from Eq. (A4) and the above relations. 
[1] G. Arcadi, M. Dutra, P. Ghosh, M. Lindner, Y. Mambrini, M. Pierre, S. Profumo, and F. S. Queiroz, Eur. Phys. J. C 78, 203 (2018).

[2] F. Zwicky, Helv. Phys. Ac. 6, 110 (1933); Gen. Relativ. Gravit. 41, 207 (2009).

[3] P. A. R. Ade et al., Astron. Astrophys. 594, A13 (2016).

[4] G. Steigman and M. S. Turner, Nucl. Phys. B253, 375 (1985).

[5] C. Patrignani et al., Chin. Phys. C 40, 100001 (2016).

[6] E. W. Varnes, Acta Phys. Pol. B 47, 1595 (2016).

[7] D. G. Charlton, Proc. Sci., ICHEP2016 (2017) 004.

[8] T. Camporesi, Proc. Sci., ICHEP2016 (2017) 005.

[9] V. Silveira and A. Zee, Phys. Lett. 161B, 136 (1985).

[10] J. McDonald, Phys. Rev. D 50, 3637 (1994).

[11] C. P. Burgess, M. Pospelov, and T. ter Veldhuis, Nucl. Phys. B619, 709 (2001).

[12] S. Kanemura, S. Matsumoto, T. Nabeshima, and N. Okada, Phys. Rev. D 82, 055026 (2010).

[13] S. Andreas, C. Arina, T. Hambye, F.-S. Ling, and M. H. G. Tytgat, Phys. Rev. D 82, 043522 (2010).

[14] A. Djouadi, O. Lebedev, Y. Mambrini, and J. Quevillon, Phys. Lett. B 709, 65 (2012).

[15] H. Zhang, Q.-H. Cao, C.-R. Chen, and C. S. Li, J. High Energy Phys. 08 (2011) 018.

[16] Y. Mambrini, Phys. Rev. D 84, 115017 (2011).

[17] A. Djouadi, A. Falkowski, Y. Mambrini, and J. Quevillon, Eur. Phys. J. C 73, 2455 (2013).

[18] S. von Buddenbrock, N. Chakrabarty, A. S. Cornell, D. Kar, M. Kumar, T. Mandal, B. Mellado, B. Mukhopadhyaya, R. G. Reed, and X. Ruan, Eur. Phys. J. C 76, 580 (2016).

[19] L. Lopez-Honorez, T. Schwetz, and J. Zupan, Phys. Lett. B 716, 179 (2012).

[20] A. Kurylov and M. Kamionkowski, Phys. Rev. D 69, 063503 (2004).

[21] J. Kearney, N. Orlofsky, and A. Pierce, Phys. Rev. D 95, 035020 (2017).

[22] G. Bambhaniya, J. Kumar, D. Marfatia, A. C. Nayak, and G. Tomar, Phys. Lett. B 766, 177 (2017).

[23] R. C. Cotta, J. L. Hewett, M. P. Le, and T. G. Rizzo, Phys. Rev. D 88, 116009 (2013).

[24] R. Bernabei et al., Eur. Phys. J. C 56, 333 (2008).

[25] E. Aprile et al., Phys. Rev. Lett. 109, 181301 (2012).
[26] C. E. Aalseth et al., Phys. Rev. D 88, 012002 (2013).

[27] E. Armengaud et al., Phys. Rev. D 86, 051701 (2012).

[28] D. S. Akerib et al., Phys. Rev. Lett. 112, 091303 (2014).

[29] R. Agnese et al., Phys. Rev. Lett. 111, 251301 (2013).

[30] R. Agnese et al., Phys. Rev. Lett. 112, 241302 (2014).

[31] G. Angloher et al., Eur. Phys. J. C 74, 3184 (2014).

[32] R. Ding and Y. Liao, J. High Energy Phys. 04 (2012) 054.

[33] K. G. Savvidy and J. D. Vergados, Phys. Rev. D 87, 075013 (2013).

[34] Z.-H. Yu, J.-M. Zheng, X.-J. Bi, Z. Li, D.-X. Yao, and H.-H. Zhang, Nucl. Phys. B860, 115 (2012).

[35] M. O. Khojali, A. Goyal, M. Kumar, and A. S. Cornell, Eur. Phys. J. C 77, 25 (2017).

[36] M. Napsuciale, S. Rodríguez, R. Ferro-Hernández, and S. Gómez-Ávila, Phys. Rev. D 93, 076003 (2016).

[37] P. Dirac, Lectures on Quantum Mechanics (Belfer Graduate School of Science, Yeshiva University, New York, 1964).

[38] S. Gómez-Ávila and M. Napsuciale, Phys. Rev. D 88, 096012 (2013).

[39] E. Delgado-Acosta, M. Kirchbach, M. Napsuciale, and S. Rodriguez, Phys. Rev. D 85, 116006 (2012).

[40] D. M. Webber et al., Phys. Rev. Lett. 106, 041803 (2011); 106, 079901 (2011).

[41] V. Khachatryan et al., J. High Energy Phys. 02 (2017) 135.

[42] S. Dodelson, Modern Cosmology (Academic Press, Amsterdam, 2003).

[43] P. Gondolo and G. Gelmini, Nucl. Phys. B360, 145 (1991).

[44] M. Cannoni, Int. J. Mod. Phys. A 32, 1730002 (2017).

[45] A. Drlica-Wagner et al., Astrophys. J. 809, L4 (2015).

[46] M. W. Goodman and E. Witten, Phys. Rev. D 31, 3059 (1985).

[47] T. M. Undagoitia and L. Rauch, J. Phys. G 43, 013001 (2016).

[48] G. Jungman, M. Kamionkowski, and K. Griest, Phys. Rep. 267, 195 (1996).

[49] E. Aprile et al., Phys. Rev. Lett. 119, 181301 (2017).

[50] E. Aprile et al., Phys. Rev. D 94, 122001 (2016).

[51] M. Cirelli, E. Del Nobile, and P. Panci, J. Cosmol. Astropart. Phys. 10 (2013) 019.

[52] P. Gondolo, J Edsjö, P Ullio, L Bergström, M Schelke, and E. A Baltz, J. Cosmol. Astropart. Phys. 07 (2004) 008. 